\title{
Ondes de choc et hypothèses de compressibilité en magnétohydrodynamique relativiste
}

\author{
ANDRÉ LICHNEROWICZ \\ Collége de France, Paris
}

Reçu le 1 Août 1968

\begin{abstract}
The principal purpose of this paper is to show that the normals to the hypersurfaces in space-time which represent the shock waves of relativistic magnetohydrodynamics are spacelike when the caloric equation of state of the medium satisfies the conditions of compressibility previously given in references [5] and [6]. Thus, the velocities of these waves are less than the local velocity of light and the theory is consistent with the postulates of a relativistic theory. A theorem previously given in reference [5] concerning the stability of Alfven shock waves, is corrected in this paper.
\end{abstract}

\section{Introduction}

Dans des travaux antérieurs $([5,6])$, nous avons étudié les ondes de choc de la magnétohydrodynamique relativiste en les supposant orientées dans le temps, donc de vitesse admissible an point de vue relativiste. Le but principal de cet article est d'abord d'établir que, sous les hypothéses de compressibilité introduites antérieurement (voir section II), les ondes de choc sont nécessairement orientées dans le temps, mais aussi de placer les vitesses de ces ondes de choc par rapport aux vitesses magnétosoniques et aux vitesses d'Alfven. L'intérêt et le caractère rigoureux des hypothéses relativistes de compressibilité se trouvent ainsi mis en pleine lumiére.

L'un des théorèmes énoncés dans [5] (p. 5l) et concernant les chocs d'Alfven est erronné. Le résultat indiqué est physiquement correct si l'on tient compte de la stabilité des ondes de choc relativement aux ondes infinitésimales d'Alfven. Les $\S 11$ et 18, 19 rectifient le point de vue et les démonstrations.

Ce mémoire est rédigé de façon à se suffire à lui-même, à quelques calculs numériques près qui figurent dans [5] et [7]. Il est divisé en six sections: I. Tenseurs-distributions et discontinuités, II. Hypothèses de compressibilité en hydrodynamique relativiste, III. Equations de la magnétohydrodynamique, IV. Ondes de choc en magnétohydrodynamique, V. Fonction d'Hugoniot et orientation des ondes de choc, VI. Thermodynamique des chocs et vitesses des ondes de choc. 


\section{Tenseurs-distributions et discontinuites}

\section{Tenseurs-distributions sur une variété riemanienne ${ }^{1}$}

a) Soit $V_{n+1}$ une variété différentiable orientée de dimension $n+1$ et classe $C^{h+1}(h \geqq 0)$; nous disposons sur $V_{n+1}$ d'une métrique riemanienne $d s^{2}$ de signature arbitraire et classe $C^{k}(0 \leqq k \leqq h)$. Localement:

$$
d s^{2}=g_{\alpha \beta} d x^{\alpha} d x^{\beta} \quad(\alpha, \beta=0,1, \ldots, n) .
$$

Si $T$ et $U$ sont deux $p$-tenseurs, nous notons $(T, U)_{x}$ le produit scalaire de $T$ et $U$ au point $x$ de $V_{n+1}$. Soit $\mathscr{D}^{p}\left(V_{n+1}\right)$ l'espace des $p$-tenseurs à support compact de classe $C^{h}$ sur $V_{n+1}$. Si $T$ est un $p$-tenseur localement sommable arbitraire, nous pouvons poser pour $U \in \mathscr{D}^{p}\left(V_{n+1}\right)$

$$
\langle T, U\rangle=\int_{V_{n+1}}(T, U)_{x} \eta(x)
$$

où $\eta$ est l'élément de volume riemannien de la variété.

Un p-tenseur-distribution $T$ de $V_{n+1}$ est une forme linéaire continue, $\grave{a}$ valeurs scalaires, sur l'espace $\mathscr{D}^{p}\left(V_{n+1}\right)$. Continu est ici entendu au sens usuel en théorie des distributions. Si $U \in \mathscr{D}^{p}\left(V_{n+1}\right),\langle T, U\rangle$ est la valeur pour $U$ du tenseur-distribution $T$.

Un $p$-tenseur localement sommable $T$ de $V_{n+1}$ peut être identifié avec un tenseur-distribution au moyen de la formule (1.1). Ce tenseur- distribution est noté $T^{D}$, ou quelquefois $T$, par abus de notation, quand aucune confusion n'est possible.

b) Nous supposons maintenant $h, k \geqq 1$.

Si $\nabla$ est l'opérateur de dérivation covariante dans la connexion riemannienne, $\delta$ l'opérateur de codérivation sur les $(p+1)$-tenseur, défini localement sur un domaine de coordonnées par

$$
\underline{\delta}: U_{\beta_{1} \ldots \beta_{p+1}} \rightarrow-\nabla_{\varrho} U_{\alpha_{1} \ldots \alpha_{p}}^{\varrho}
$$

la dérivée covariante $\nabla T$ d'un p-tenseur distribution $T$ est définie naturellement comme le $(p+1)$-tenseur distribution satisfaisant la relation

$$
\langle\nabla T, U\rangle=\langle T, \underline{\delta} U\rangle \quad\left(U \in \mathscr{D}^{\varrho}\left(V_{n+1}\right)\right) .
$$

Il est aisé de voir que toutes les propriétés classiques de la dérivée covariante dans une connexion riemannienne, ainsi que les formules correspondantes, deneurent valables pour les tenseurs-distributions.

c) Nous considérons maintenant exclusivement un domaine $\Omega$ de $V_{n+1}$. Soit $\Sigma$ une hypersurface régulière définie par l'équation locale $\varphi=0\left(\varphi\right.$ de classe $\left.C^{2}\right)$ qui partage $\Omega$ en deux domaines $\Omega_{0}$ et $\Omega_{1}$ correspondant respectivement à $\varphi<0$ et $\varphi>0$. Nous notons par $l \neq 0$ le gradient de $\varphi$.

Considérons la classe des $n$-formes $\omega$ vérifiant la relation:

$$
\eta=d \varphi \wedge \omega=l \wedge \omega .
$$

\footnotetext{
1 Voir par exemple LICHNEROwicz [4].
} 
Si $\omega$ et $\omega^{\prime}$ sont deux formes de cette classe, il existe une $(n-1)$-forme $\mu$ telle que $\omega^{\prime}=\omega+d \varphi \wedge \mu$. Soit $\partial \Omega_{0}$ et $\partial \Omega_{1}$ les bords orientés sur $\Sigma$ de $\Omega_{0}$ et $\Omega_{1}\left(\partial \Omega_{0}=-\partial \Omega_{1}\right)$. D'après la remarque précédente, l'intégrale

$$
\int_{\partial \Omega_{0}} f \omega=-\int_{\partial \Omega_{1}} f \omega, \quad\left(f \in \mathscr{D}^{\circ}(\Omega)\right)
$$

a une valeur bien déterminée indépendante du choix de $\omega$ dans la classe envisagée. Nous pouvons ainsi définir un scalaire distribution $\bar{\delta}_{\varphi}$ (ou plus brièvement $\bar{\delta}$ ) par la relation:

$$
\langle\bar{\delta}, f\rangle=\int_{\partial \Omega_{0}} f \omega=-\int_{\partial \Omega_{1}} f \omega, \quad\left(f \in \mathscr{D}^{\circ}(\Omega)\right)
$$

$\bar{\delta}_{\varphi}$ est la mesure de Dirac relative à $\varphi$; son support est porté par $\Sigma$.

\section{Tenseurs-discontinuités à la traversée d'une hypersurface}

a) Considérons un $p$-tenseur $T$ sur $\Omega$ satisfaisant les hypothèses suivantes:

$\mathrm{A}_{1}$ ) Sur chacun des domaines $\Omega_{0}$ et $\Omega_{1}$, le tenseur $T$ est un tenseur ordinaire de classe $C^{1}$.

$\mathrm{A}_{2}$ ) Quand $\varphi$ tend vers zéro par valeurs négatives (resp. positives), $T$ et $\nabla T$ convergent uniformément vers des fonctions à valeurs tensorielles définies sur $\Sigma$ et notées $T_{0},(\nabla T)_{0}\left(\operatorname{resp} . T_{1},(\nabla T)_{1}\right)$.

Nous introduisons les tenseurs-discontinuités sur $\Sigma$ :

$$
[T]=T_{1}-T_{0}, \quad[\nabla T]=(\nabla T)_{1}-(\nabla T)_{0}
$$

Si $T^{D}$ est le tenseur-distribution défini par le tenseur $T$, le tenseurdistribution $\nabla T^{D}$, dérvée au sens des distributions de $T^{D}$ est donné par la formule [7]

$$
\nabla T^{D}=l \bar{\delta}[T]+(\nabla T)^{D}
$$

Dans cette formule, le terme $(\nabla T)^{D}$ est le tenseur-distribution défini par le tenseur ordinaire localement sommable $\nabla T$, dérivée covariante usuelle du tenseur $T$. Le terme $l \bar{\delta}[T]$ est appelé la O-couche tensorielle correspondant au tenseur-discontinuité $[T]$ sur $\Sigma$.

En étudiant la dérivée du tenseur-distribution $\bar{\delta}[T]$ de $\Omega$ ([7]), on voit qu'il existe un $p$-tenseur distribution, noté $\delta T$, tel que l'on ait:

$\delta T$ a pour support $\Sigma$.

$$
\bar{\delta}[\nabla T]=\nabla(\bar{\delta}[T])+l \delta T
$$

c) Nous considérons maintenant des tenseurs satisfaisant toujours aux hypothèses $A_{1}, A_{2}$ mais qui sont supposés continus sur $\Omega$. Ces tenseurs définissent d'une manière naturelle une algèbre $\mathscr{A}$ de tenseurs. La formule (2.2) devient alors:

Considérons l'application :

$$
\bar{\delta}\left[\nabla_{\alpha} T\right]=l_{\alpha} \cdot \delta T .
$$

$$
\delta: T \in \mathscr{A} \rightarrow \delta T
$$


où $\delta T$ est un tenseur-distribution à support sur $\Sigma$. On déduit de (2.3) que l'application $\delta$ est une dérivation: si $a$ et $b$ sont deux réels et si $T, U \in \mathscr{A}$ sont deux $p$-tenseurs, il résulte de (2.3):

$$
\delta(a T+b U)=a \delta T+b \delta U .
$$

Si $T, U \in \mathscr{A}$ sont respectivement un $p$-tenseur et un $q$-tenseur, on $a$ :

$$
\delta(T \otimes U)=\delta T \otimes U+T \otimes \delta U
$$

$\delta T$ est appelé la discontinuité infinitésimale de $T$ et $\delta$ l'opérateur de discontinuité infinitésimale.

\section{Hypotheses de compressibilité en hydrodynamique relativiste}

\section{Fluide parfait thermodynamique relativiste}

a) Soit $V_{4}$ un espace-temps muni d'une métrique hyperbolique $d s^{2}$, de signature + - - , satisfaisant aux hypothèses de différentiabilité de I. Dans $V_{4}$ un fluide parfait est décrit par un tenseur d'énergie

$$
T_{\alpha \beta}^{(f)}=(\varrho+p) u_{\alpha} u_{\beta}-p g_{\alpha \beta}, \quad(\alpha, \beta=0,1,2,3)
$$

où $\varrho$ est la densité propre d'énergie du fluide, $p$ la pression, $u_{\alpha}$ le vecteurvitesse unitaire du fluide orienté vers le futur, $g_{\alpha \beta}$ le tenseur métrique. La densité d'énergie $\varrho$ se compose de la densité propre de matière et de la densité d'énergie interne du fluide. En accord avec TaUb [8], nous posons

$$
\varrho=c^{2} r\left(1+\frac{\varepsilon}{c^{2}}\right) \quad(r, \varepsilon>0) .
$$

où $r$ est la densité propre de matière et $\varepsilon$ l'énergie interne spécifique du fluide. Par définition la densité de matière $r$ est conservative au cours du mouvement (conservation du nombre de particles). Si $\nabla$ est l'opérateur de dérivation covariante, $r$ est supposée satisfaire

$$
\nabla_{\alpha}\left(r u^{\alpha}\right)=0 .
$$

Dans (3.1) apparaît le scalaire:

Nous posons:

$$
\varrho+p=c^{2} r\left(1+\frac{\varepsilon}{c^{2}}+\frac{p}{c^{2} r}\right) .
$$

$$
V=\frac{1}{r}, \quad i=\varepsilon+\frac{p}{r}=\varepsilon+p V
$$

où $V$ est le volume spécifique et $i$ l'enthalpie spécifique. A cette enthalpie, il est préférable, dans le cadre relativiste, de substituer ce que j'ai appelé l'indice du fluide

$$
f=1+\frac{i}{c^{2}}, \quad(f \geqq 1)
$$


qui se réduit à l'unité à l'approximation classique. Le tenseur d'énergie (3.1) s'écrit alors:

$$
T_{\alpha \beta}^{(f)}=c^{2} r f u_{\alpha} u_{\beta}-p g_{\alpha \beta} .
$$

b) La température propre $\Theta$ du fluide et son entropie spécifique $S$ peuvent être définies comme en hydrodynamique classique, par la relation différentielle

$$
\Theta d S=d \varepsilon+p d V=d i-V d p=c^{2} d f-V d p \quad(\Theta>0) .
$$

On en déduit:

$$
c^{2} d f=V d p+\Theta d S
$$

La variable thermodynamique $\tau=f V$ joue dans le cadre relativiste un rôle important et se substitue le plus souvent au volume spécifique classique. Il est commode d'adopter $p$ et $S$ comme variables thermodynamiques de base. Nous considérons $\tau=\tau(p, S)$ comme une fonction donnée définissant, pour le fluide, une équation d'état.

c) Le système différentiel fondamental de l'hydrodynamique est fourni par les relations de conservation:

$$
\nabla_{\alpha}\left(r u^{\alpha}\right)=0, \quad \nabla_{\alpha} T^{(f) \alpha \beta}=0 .
$$

On vérifie immédiatement qu'elles entraînent:

$$
u^{\alpha} \partial_{\alpha} S=0
$$

et fournissent le système différentiel aux lignes de courant:

$$
c^{2} r f u^{\alpha} \nabla_{\alpha} u^{\beta}-\left(g^{\alpha \beta}-u^{\alpha} u^{\beta}\right) \partial_{\alpha} p=0 .
$$

Inversement (3.2), (3.6), (3.7) forment un système équivalent à (3.5).

4. Vitesse d'une hypersurface par rapport au fluide et ondes soniques

a) Soit $\Sigma$ une hypersurface régulière de $V_{4}$ d'équation locale $\varphi=0$ (avec $l=d \varphi$ ). La vitesse $v^{\Sigma}$ de l'hypersurface $\Sigma$ par rapport au fluide est donnée par la formule

$$
\frac{\left(v^{\Sigma}\right)^{2}}{c^{2}}=y^{\Sigma} \quad \text { avec } \quad y^{\Sigma}=\frac{\left(u^{\alpha} l_{\alpha}\right)^{2}}{\left(u^{\alpha} u^{\beta}-g^{\alpha \beta}\right) l_{\alpha} l_{\beta}}=\frac{\left(u^{\alpha} l_{\alpha}\right)^{2}}{\left(u^{\alpha} l_{\alpha}\right)^{2}-l^{\alpha} l_{\alpha}} .
$$

On voit que, quel que soit $l, y^{\Sigma}$ est positif et que l'on a:

$$
l^{\alpha} l_{\alpha}>0 \Leftrightarrow y^{\Sigma}>1 ; \quad l^{\alpha} l_{\alpha}=0 \Leftrightarrow y^{\Sigma}=1 ; \quad l^{\alpha} l_{\alpha}<0 \Leftrightarrow y^{\Sigma}<1 .
$$

b) Dans un domaine $\Omega$ où les variables thermodynamiques $p$, $S$ et le vecteur-vitesse $u^{\beta}$ sont continus, supposons que les hypothèses $A_{1}, A_{2}$ soient satisfaites par $p, S, u^{\beta}$ relativement à une hypersurface $\Sigma$, les dérivées premières étant discontinues. 
D'après (2.3) il existe des distributions à support sur $\Sigma$ notées $\delta p$, $\delta S, \delta u^{\beta}$ telles que:

$$
\bar{\delta}\left[\nabla_{\alpha} p\right]=l_{\alpha} \delta p, \quad \bar{\delta}\left[\nabla_{\alpha} S\right]=l_{\alpha} \delta S, \quad \bar{\delta}\left[\nabla_{\alpha} u^{\beta}\right]=l_{\alpha} \delta u^{\beta}
$$

où $\bar{\delta}$ est relative à $\varphi$. L'étude des conditions pour que l'une au moins des distributions $\delta p, \delta S, \delta u^{\beta}$ ne soit pas nulle conduit aux deux cas d'hypersurfaces caractéristiques du système différentiel (3.5) (voir [6]).

$1^{\circ}$ ) Des hypersurfaces engendrées par des lignes de courant, vérifiant

$$
u^{\alpha} l_{\alpha}=0 \text {. }
$$

Ce sont les ondes de matière (ou d'entropie). Leur vitesse par rapport au fluide est nulle.

$2^{\circ}$ ) Des hypersurfaces vérifiant l'équation:

$$
P(l) \equiv\left(g^{\alpha \beta}-u^{\alpha} u^{\beta}\right) l_{\alpha} l_{\beta}+\gamma\left(u^{\alpha} l_{\alpha}\right)^{2}=0
$$

où $\gamma$ est donnée à partir de l'équation d'état par la relation:

$$
c^{2} \tau_{p}^{\prime}=-V^{2}(\gamma-1) \text {. }
$$

Ce sont les ondes soniques. Si $v$ est la vitesse des ondes soniques par rapport au fluide, on déduit de (4.3)

$$
\frac{v^{2}}{c^{2}}=\frac{1}{\gamma}\left(=y^{\Sigma}\right) \text {. }
$$

Nous postulons dans la suite que $v<c$ (ou $\gamma>1$ ), ce qui revient d'après le a à postuler que les ondes soniques sont orientées dans le temps. Pour que $v$ soit $<c$, il faut et il suffit, d'après (4.4), que $\tau_{p}^{\prime}$ soit $<0$.

\section{Hypothèses de compressibilité}

a) On a été conduit (voir [5], [3]) à adopter pour les fluides parfaits relativistes les hypothèses de compressibilité suivantes portant sur la fonction $\tau(p, S)$

$$
\tau_{p}^{\prime}<0, \quad \tau_{S}^{\prime}>0
$$

et la condition de convexité

$$
\tau_{p^{2}}^{\prime \prime}>0
$$

Nous avons vu que l'inégalité $\tau_{p}^{\prime}<0$ exprime que la vitesse sonique $v$ du fluide est inférieure à $c$ (où que les ondes soniques sont orientées dans le temps). Les hypothèses de compressibilité $\left(\mathrm{H}_{1}\right),\left(\mathrm{H}_{2}\right)$ se réduisent à l'approximation classique aux hypothèses usuelles, dites de HERmaN WEYL, concernant la fonction $V(p, S)$. Ces hypothèses sont satisfaites par les gaz polytropiques relativistes [5]; des hypothèses semblables ont été étudiées par ISRAEL [3].

b) En inversant la fonction $\tau=\tau(p, S)$, on obtient une fonction $S=S(p, \tau)$ exprimant l'entropie en fonction des variables $p$ et $\tau$. On 
a identiquement en $p$ et $S$ :

$$
S=S\{p, \tau(p, S\} .
$$

Nous nous proposons de traduire les hypothèses de compressibilité faites en termes de la fonction $S(p, \tau)$. Par dérivation de (5.1) par rapport à $S$ à $p$ constant, il vient:

On en déduit:

$$
S_{\tau}^{\prime} \tau_{S}^{\prime}=1
$$

$$
S_{\tau}^{\prime}=\frac{1}{\tau_{S}^{\prime}}
$$

De même, par dérivation de (5.1) par rapport à $p$, à $S$ constant, on a:

Il en résulte

$$
S_{p}^{\prime}+S_{\tau}^{\prime} \tau_{p}^{\prime}=0
$$

$$
S_{p}^{\prime}=-\frac{\tau_{p}^{\prime}}{\tau_{S}^{\prime}}
$$

Les hypothèses de compressibilité $\left(\mathrm{H}_{1}\right)$ se traduisent donc par les inégalités :

$$
S_{p}^{\prime}>0, \quad S_{\tau}^{\prime}>0 .
$$

c) En dérivant (5.3) par rapport à $p$, à $S$ constant, il vient:

On en déduit:

$$
S_{p^{2}}^{\prime \prime}+2 S_{p \tau}^{\prime \prime} \tau_{p}^{\prime}+S_{\tau^{2}}^{\prime \prime}\left(\tau_{p}^{\prime}\right)^{2}+S_{\tau}^{\prime} \tau_{p^{2}}^{\prime \prime}=0
$$

$$
\left(S_{\tau}^{\prime}\right)^{3} \tau_{p^{2}}^{\prime \prime}=-\left\{S_{p^{2}}^{\prime \prime}\left(S_{\tau}^{\prime}\right)^{2}-2 S_{p \tau}^{\prime \prime} S_{p}^{\prime} S_{\tau}^{\prime}+S_{\tau^{2}}^{\prime \prime}\left(S_{p}^{\prime}\right)^{2}\right\}
$$

Sous l'hypothèse $\tau_{s}^{\prime}>0$, l'hypothèse $\left(\mathrm{H}_{2}\right)$ se traduit donc par l'inégalité:

$$
S_{p^{2}}^{\prime \prime}\left(S_{\tau}^{\prime}\right)^{2}-2 S_{p \tau}^{\prime \prime} S_{p}^{\prime} S_{\tau}^{\prime}+S_{\tau^{2}}^{\prime \prime}\left(S_{p}^{\prime}\right)^{2}<0
$$

\section{Les equations de la magnétohydrodynamique}

\section{Le tenseur d'énergie de la magnétohydrodynamique relativiste}

a) Supposons le fluide envisagé soumis à un champ électromagnétique décrit par deux tenseurs dont l'un $H$ est le tenseur champ électriqueinduction magnétique. Si * est l'opérateur d'adjonction sur les tenseurs antisymétriques, les vecteurs orthogonaux à $u$, donc spatiaux,

$$
e_{\beta}=u^{\alpha} H_{\alpha \beta}, \quad b_{\beta}=u^{\alpha}(* H)_{\alpha \beta}
$$

sont respectivement le vecteur champ électrique et le vecteur induction magnétique relatifs à la direction temporelle $u$. Soit $\mu$, constante donnée, la perméabilité magnétique du fluide. Le vecteur champ magnétique $h$ est supposé relié à l'induction magnétique $b$ par la relation $b_{\beta}=\mu h_{\beta}$.

Le courant électrique $J$ est sensiblement la somme de deux termes

$$
J^{\beta}=v u^{\beta}+\sigma e^{\beta}
$$

où $\nu$ est la densité propre de charge électrique et $\sigma$ la conductivité du fluide. 
b) La magnétohydrodynamique est ici l'étude des propriétés d'un fluide relativiste de conductivité infinie; $J$ étant essentiellement fini, il en est de même pour $\sigma e$ et nécessairement $e=0$. Par rapport à la direction temporelle u définie par la vitesse du fluide, le champ électromagnétique se réduit à sa partie magnétique.

D'après des résultats classiques, ce champ admet le tenseur d'énergie:

$$
\tau_{\alpha \beta}=\mu\left\{|h|^{2}\left(u_{\alpha} u_{\beta}-\frac{1}{2} g_{\alpha \beta}\right)-h_{\alpha} h_{\beta}\right\}
$$

où $|h|^{2}=-h^{\circ} h_{\varrho}$ est strictement positif pour $h_{\varrho} \neq 0$. Le tenseur d'énergie total s'en déduit par addition de (3.3):

$T_{\alpha \beta}=\left(c^{2} r f+\mu|h|^{2}\right) u_{\alpha} u_{\beta}-q g_{\alpha \beta}-\mu h_{\alpha} h_{\beta} \quad$ avec $q=p+\frac{1}{2} \mu|h|^{2}$

\section{\%. Le système différentiel fondamental de la magnétohydrodynamique}

Ce système est fourni par les considérations suivantes: nous postulons encore que $r$ vérifie:

$$
\nabla_{\alpha}\left(r u^{\alpha}\right)=0 .
$$

Les équations de MAxwell se réduisent ici à:

$$
\nabla_{\alpha}\left(h^{\alpha} u^{\beta}-u^{\alpha} h^{\beta}\right)=0 .
$$

Les équations de la dynamique relativiste sont fournies par la conservation du tenseur d'énergie donné par (6.1):

$$
\nabla_{\alpha} T^{\alpha \beta}=0 .
$$

Le système (7.1), (7.2), (7.3) constitue le système fondamental de la magnétohydrodynamique. On vérifie immédiatement qu'il entraîne encore

$$
u^{\alpha} \partial_{\alpha} S=0
$$

et fournit le système différentiel aux lignes de courant:

$$
\begin{aligned}
& \left(c^{2} r f+\mu|h|^{2}\right) u^{\alpha} \nabla_{\alpha} u^{\beta}-\left(g^{\alpha \beta}-u^{\alpha} u^{\beta}\right) \partial_{\alpha} q \\
& \quad+\mu\left(\frac{1}{2} u^{\alpha} \partial_{\alpha}|h|^{2}+|h|^{2} \nabla_{\alpha} u^{\alpha}\right) u^{\beta}-\mu \nabla_{\alpha} h^{\alpha} h^{\beta}-\mu h^{\alpha} \nabla_{\alpha} h^{\beta}=0 .
\end{aligned}
$$

Inversement (7.1), (7.2), (7.4), (7.5) forment un système équivalent au système fondamental. On peut encore noter que ces relations entraînent [6]

$$
\nabla_{\alpha}\left(f h^{\alpha}\right)-\frac{1}{c^{2}} \Theta h^{\alpha} \partial_{\alpha} S=0 .
$$

\section{Ondes magnétosoniques et ondes d'Alfven}

a) Dans un domaine $\Omega$ où les variables thermodynamiques $p, S$ et les vecteurs $u^{\beta}, h^{\beta}$ sont continus, supposons que les hypothèses $A_{1}, A_{2}$ 
soient satisfaites par ces grandeurs, relativement à une hypersurface $\Sigma$ (d'équation $\varphi=0$, avec $l=d \varphi$ ), les dérivées premières étant discontinues.

D'après (2.3), il existe des distributions à support sur $\Sigma$ notées $\delta p$, $\delta S, \delta u^{\beta}, \delta h^{\beta}$ telles que:

$\bar{\delta}\left[\nabla_{\alpha} p\right]=l_{\alpha} \delta p, \quad \bar{\delta}\left[\nabla_{\alpha} S\right]=l_{\alpha} \delta S, \quad \bar{\delta}\left[\nabla_{\alpha} u^{\beta}\right]=l_{\alpha} \delta u^{\beta} \quad \bar{\delta}\left[\nabla_{\alpha} h^{\beta}\right]=l_{\alpha} \delta h^{\beta}$

L'étude des conditions pour que l'une au moins des distributions $\delta p$, $\delta S, \delta u^{\beta}, \delta h^{\beta}$ ne soit pas nulle conduit aux trois cas d'hypersurfaces caractéristiques du système différentiel fondamental $[6]$ :

$\left.1^{\circ}\right)$ Des hypersurfaces engendrées par des lignes de courant, vérifient:

$$
u^{\alpha} l_{\alpha}=0
$$

ou ondes de matière, à vitesse nulle par rapport au fluide.

$2^{\circ}$ ) Des hypersurfaces vérifiant l'équation:

$$
\begin{gathered}
P(l) \equiv c^{2} r f(\gamma-1)\left(u^{\alpha} l_{\alpha}\right)^{4}+\left(c^{2} r f+\mu|h|^{2} \gamma\right)\left(u^{\alpha} l_{\alpha}\right)^{2} l^{\beta} l_{\beta} \\
-\mu\left(h^{\alpha} l_{\alpha}\right)^{2} l^{\beta} l_{\beta}=0 .
\end{gathered}
$$

Ce sont les ondes magnétosoniques qui correspondent à l'existence de discontinuités des dérivées de $p$ et des composantes normales à $\Sigma$ de $u^{\beta}$ et $h^{\beta}$.

$3^{\circ}$ ) Des hypersurfaces vérifiant l'équation:

$$
D(l) \equiv\left(c^{2} r f+\mu|h|^{2}\right)\left(u^{\alpha} l_{\alpha}\right)^{2}-\mu\left(h^{\alpha} l_{\alpha}\right)^{2}=0 .
$$

Ce sont les ondes d'Alfven qui correspondent, en l'absence d'autres discontinuités, à l'existence de discontinuités des dérivées des composantes tangentielles de $u^{\beta}$ et $h^{\beta}$.

b) Posons pour abréger

$$
\beta=\sqrt{c^{2} r f+\mu|h|^{2} / \mu} .
$$

L'équation (8.2) aux ondes d'Alfven peut s'écrire

$$
\left\{\left(\beta u^{\alpha}+h^{\alpha}\right) l_{\alpha}\right\}\left\{\left(\beta u^{\beta}-h^{\beta}\right) l_{\beta}\right\}=0
$$

et l'on voit que les ondes d'Alfven sont engendrées par les trajectoires des champs de vecteurs temporels

$$
A^{\alpha}=\beta u^{\alpha}+h^{\alpha}, \quad B^{\alpha}=\beta u^{\alpha}-h^{\alpha}
$$

ce qui définit deux types d'ondes dites $A$ ou $B$.

c) J'ai établi (voir [6]) pour le système de la magnétohydrodynamique, le premier théorème local d'existence et d'unicité du problème de Cauchy sur des classes de fonctions $C^{\infty}$ (classes de Gevrey) et montré que, bien que le système ne soit pas strictement hyperbolique au sens de GARDING-Leray, il y a cependant domaine d'influence. 
9. Vitesses des ondes magnétosoniques et des ondes d'Alfven

A toute hypersurface $\Sigma$ (d'équation $\varphi=0$, avec $l=d \varphi$ ), nous avons associé (\$4) le paramètre:

$$
y^{\Sigma}=\frac{\left(u^{\alpha} l_{\alpha}\right)^{2}}{\left(u^{\alpha} l_{\alpha}\right)^{2}-l^{\alpha} l_{\alpha}} \geqq 0 .
$$

La relation (9.1) est équivalente à la relation:

$$
\left(1-y^{\Sigma}\right)\left(u^{\alpha} l_{\alpha}\right)^{2}=-\left(l^{\alpha} l_{\alpha}\right) y^{\Sigma} \text {. }
$$

a) De manière analogue, la composante $h_{n}$ du champ magnétique définie par:

$$
h_{n}^{2}=\frac{\left(h^{\alpha} l_{\alpha}\right)^{2}}{\left(u^{\alpha} u^{\beta}-g^{\alpha \beta}\right) l_{\alpha} l_{\beta}}=\frac{\left(h^{\alpha} l_{\alpha}\right)^{2}}{\left(u^{\alpha} l_{\alpha}\right)^{2}-l^{\alpha} l_{\alpha}} \geqq 0
$$

vérifie le lemme suivant:

Lemme 1. On a toujours $h_{n}^{2} \leqq|h|^{2}$.

2. Pour que $h_{n}^{2}=|h|^{2}$, il faut et il suffit que $l$ appartienne au 2-plan défini par $(u, h)$.

En effet considérons un repère orthonormé $\left(e_{0}, e_{i}\right)(i, j=1,2,3)$ en $x \in V_{4}$ tel que $e_{0}=u$. On a $h^{0}=0$ et il vient d'après l'inégalité de ScHwarz

soit

$$
\left(h^{i} l_{i}\right)^{2} \leqq \sum_{i}\left(h^{i}\right)^{2} \cdot \sum_{i}\left(l^{i}\right)^{2}
$$

$$
\frac{\left(h^{i} l_{i}\right)^{2}}{\Sigma\left(l^{i}\right)^{2}} \leqq|h|^{2} \quad \text { ou } \quad h_{n}^{2} \leqq|h|^{2}
$$

ce qui établit le $\mathrm{I}^{\circ}$. Pour que l'égalité ait lieu, il faut et il suffit que si nous décomposons $l$ selon $u$ et un vecteur $k$ orthogonal, $k$ soit colinéaire à $h$, ce qui démontre la seconde partie du lemme.

b) En introduisant $h_{n}^{2}$, la quantité $P(l)$ peut s'écrire:

$$
\begin{aligned}
P(l) \equiv c^{2} r f(\gamma-1)\left(u^{\alpha} l_{\alpha}\right)^{4} & +\left(c^{2} r f+\mu|h|^{2} \gamma-\mu h_{n}^{2}\right) \cdot\left(u^{\alpha} l_{\alpha}\right)^{2} l^{\beta} l_{\beta} \\
& +\mu h_{n}^{2}\left(l^{\beta} l_{\beta}\right)^{2} .
\end{aligned}
$$

D'après (9.2), $P(l)$ peut s'exprimer en termes de $y^{\Sigma}$ par:

où

$$
\frac{\left(1-y^{\Sigma}\right)^{2} P(l)}{\left(l^{\beta} l_{\beta}\right)^{2}}=\Pi\left(y^{\Sigma}\right)
$$

$\Pi(y)=c^{2} r f(\gamma-1) y^{2}+\left(c^{2} r f+\mu|h|^{2} \gamma-\mu h_{n}^{2}\right) y(1-y)+\mu h_{n}^{2}(1-y)^{2}$ c'est-à-dire en développant et ordonnant en $y$ :

$$
\Pi(y)=\left(c^{2} r f+\mu|h|^{2}\right) \gamma y^{2}-\left(c^{2} r f+\mu|h|^{2} \gamma+\mu h_{n}^{2}\right) y+\mu h_{n}^{2}
$$

Sous la seule hypothèse $\tau_{p}^{\prime}<0$ (c'est-à-dire $\gamma>1$ ), le trinôme $\Pi(y)$ a les propriétés suivantes

$$
\Pi(0)=\mu h_{n}^{2} \geqq 0, \quad \Pi(1)=c^{2} r f(\gamma-1)>0
$$


et

$$
\Pi\left(\frac{v^{2}}{c^{2}}\right)=\Pi\left(\frac{1}{\gamma}\right)=\mu\left(|h|^{2}-h_{n}^{2}\right)\left(\frac{1}{\gamma}-1\right) \leqq 0 .
$$

Ainsi $\Pi(y)$ a deux zéros entre 0 et 1 que nous désignons par $y^{M L}$ et $y^{M R}$. On en déduit qu'il existe pour les ondes magnétosoniques deux vitesses $v^{M L}$ et $v^{M R}$ par rapport au fluide définies par:

$$
\left(v^{M L}\right)^{2} / c^{2}=y^{M L}, \quad\left(v^{M R}\right)^{2} / c^{2}=y^{M R}
$$

et vérifiant les inégalités:

$$
v^{M L} \leqq v \leqq v^{M R}<c
$$

$v^{M L}$ est appelée la vitesse des ondes magnétosoniques lentes et $v^{M L}$ la vitesse des ondes magnétosoniques rapides.

c) En introduisant $h_{n}^{2}$ dans $D(l)$, il vient:

$$
\frac{D(l)}{\left(c^{2} r f+\mu|h|^{2}\right)\left(\left(u^{\alpha} l_{\alpha}\right)^{2}-l^{\alpha} l_{\alpha}\right)}=y^{\Sigma}-\frac{\mu h_{n}^{2}}{c^{2} r f+\mu|h|^{2}} .
$$

Ainsi les ondes d'Alfven admettent par rapport au fluide une vitesse $v^{A}$ donnée par:

$$
\frac{\left(v^{A}\right)^{2}}{c^{2}}=\frac{\mu h_{n}^{2}}{c^{2} r f+\mu|h|^{2}}<1 .
$$

Un calcul immédiat donne:

$$
\Pi\left(\frac{\left(v^{A}\right)^{2}}{c^{2}}\right)=\frac{\left(v^{A}\right)^{2}}{c^{2}} \mu\left(h_{n}^{2}-|h|^{2}\right)(\gamma-1) \leqq 0 .
$$

On voit ainsi que:

$$
v^{M L} \leqq v^{A} \leqq v^{M R}
$$

\section{Ondes de choc en magnétohydrodynamique relativiste}

10. Le système fondamental des ondes de choc

a) Dans un domaine $\Omega$ de $V_{4}$, soit encore $\Sigma$ une hypersurface d'équation locale $\varphi=0$ (avec $l=d \varphi$ ). L'hypersurface $\Sigma$ est une onde de choc magnétohydrodynamique si $u^{a}, h^{a}$ ou l'une au moins des variables thermodynamiques est discontinu à la traversée de $\Sigma$.

Au voisinage de $\Sigma, p, S, u^{a}, h^{a}$ sont supposés vérifier les hypothèses $A_{1}$ et $A_{2}$. Ainsi

$1^{\circ}$ ) Sur chacun des domaines $\Omega_{0}$ et $\Omega_{1}, p, S, u^{a}, h^{a}$ sont de classe $C^{1}$.

$2^{\circ}$ ) Quand $\varphi$ tend vers zéro par valeurs négatives (resp. positives), ils convergent uniformément, ainsi que leurs dérivées covariantes, vers des fonctions tensorielles définies sur $\Sigma$.

Les notations sont celles du $\S 2$. Nous supposons que le système fondamental (7.1), (7.2), (7.3) de la magnétohydrodynamique est satisfait au sens des distributions:

$$
\nabla_{\alpha}\left(r u^{\alpha}\right)^{D}=0, \quad \nabla_{\alpha}\left(h^{\alpha} u^{\beta}-u^{\alpha} h^{\beta}\right)^{D}=0, \quad \nabla_{\alpha}\left(T^{\alpha \beta}\right)^{D}=0 .
$$


En introduisant des éléments d'essai à supports compacts dans $\Omega_{0}$ (resp. $\Omega_{1}$ ) on voit que ces relations entraînent bien sur $\Omega_{0}$ (resp. $\Omega_{1}$ )

$$
\nabla_{\alpha}\left(r u^{\alpha}\right)=0, \quad \nabla_{\alpha}\left(h^{\alpha} u^{\beta}-u^{\alpha} h^{\beta}\right)=0, \quad \nabla_{\alpha}\left(T^{\alpha \beta}\right)=0
$$

ce qui implique:

$$
\left(\nabla_{\alpha}\left(r u^{\alpha}\right)\right)^{D}=0, \quad\left(\nabla_{x}\left(h^{\alpha} u^{\beta}-u^{\alpha} h^{\beta}\right)\right)^{D}=0, \quad\left(\nabla_{\alpha} T^{\alpha \beta}\right)^{D}=0 .
$$

De (10.1) et (10.2), il résulte d'après la formule (2.1):

$$
l_{\alpha}\left[r u^{\alpha}\right]=0, \quad l_{\alpha}\left[h^{\alpha} u^{\beta}-u^{\alpha} h^{\beta}\right]=0, \quad l_{\alpha}\left[T^{\alpha \beta}\right]=0 .
$$

Le système (10.3) est le système fondamental des ondes de choc.

Nous établirons que sous les hypothèses de compressibilité $\left(\mathrm{H}_{1}\right), \Sigma$ est nécessairement orientée dans le temps, donc de vitesse admissible au point de vue relativiste.

b) Soit $Y$ un état du fluide défini par les valeurs en un point $x$ de $\Sigma$ de $p, S, u^{a}, h^{a}$. Un tel état est défini par la donnée de 8 composantes. En décomposant $u^{\beta}$ et $h^{\beta}$ selon leurs composantes normales et tangentes à $\Sigma$, on a:

$$
u^{\beta}=v^{\beta}+\frac{u^{\alpha} l_{\alpha}}{l^{\alpha} l_{\alpha}} l^{\beta}, \quad h^{\beta}=t^{\beta}+\frac{h^{\alpha} l_{\alpha}}{l^{\alpha} l_{\alpha}} l, \quad\left(v^{\beta} l_{\beta}=0, \quad t^{\beta} l_{\beta}=0\right) .
$$

Posons

$$
a(Y)=r u^{\alpha} l_{\alpha}, \quad V^{\beta}(Y)=\left(h^{\alpha} l_{\alpha}\right) u^{\beta}-\frac{a(Y)}{r} h^{\beta}
$$

et

$$
W^{\beta}(Y)=\left(c^{2} \tau+\mu \frac{|h|^{2}}{r^{2}}\right) a(Y) r u^{\beta}-q l^{\beta}-\mu\left(h^{\alpha} l_{\alpha}\right) h^{\beta} .
$$

Il est clair que le vecteur $V^{\beta}$ est tangent à $\Sigma$. Si $Y_{0}$ et $Y_{1}$ désignent les états respectivement antérieur et postérieur au choc, le système (10.3) exprime que

$$
a\left(Y_{1}\right)=a\left(Y_{0}\right), \quad V^{\beta}\left(Y_{1}\right)=V^{\beta}\left(Y_{0}\right), \quad W^{\beta}\left(Y_{1}\right)=W^{\beta}\left(Y_{0}\right) .
$$

Le scalaire $a$ et les vecteurs $V^{\beta}$ et $W^{\beta}$ définissent les invariants du choc.

Un choc est dit tangentiel si $a=0$. On a alors $u_{0}^{\alpha} l_{\alpha}=u_{1}^{\alpha} l_{\alpha}=0 ; \Sigma$ est de vitesse nulle par rapport au fluide dans les deux états, donc orientée dans le temps. Les chocs tangentiels ont été étudiés ailleurs [5]. Nous les écartons dans la suite et supposons $a \neq 0$.

c) Introduisons le scalaire invariant

$$
H=\frac{1}{a^{2}} V^{\beta} V_{\beta}=\frac{\left(h^{\alpha} l_{\alpha}\right)^{2}}{a^{2}}-\frac{|h|^{2}}{r^{2}} .
$$

En substituant dans $W^{\beta}$ à $h^{\beta}$ son expression en termes de $V^{\beta}$ et $u^{\beta}$, il vient:

$$
W^{\beta}=a \alpha r u^{\beta}-q l^{\beta}+\mu \frac{r}{a}\left(h^{\alpha} l_{\alpha}\right) V^{\beta}
$$


où l'on a introduit la variable importante:

$$
\alpha=c^{2} \tau-\mu H=\frac{D(l)}{a^{2}} .
$$

Si nous décomposons $W^{\beta}$ en sa partie tangentielle et sa partie normale, on obtient:

où

$$
W^{\beta}=X^{\beta}-\left(q-\frac{a^{2}}{l^{\alpha} l_{\alpha}} \alpha\right) l^{\beta}
$$

$$
X^{\beta}=a \alpha r v^{\beta}+\mu \frac{a}{r}\left(h^{\alpha} l_{\alpha}\right) V^{\beta}
$$

est tangent à $\Sigma$. L'invariance de $W^{\beta}$ est équivalente à l'ensemble de celle de $X^{\beta}$ et de celle de la composante normale. Ainsi le scalaire:

est invariant.

$$
e=q-\frac{c^{2} a^{2}}{l^{\alpha} l_{\alpha}} \tau
$$

Considérons en particulier le produit scalaire invariant au cours $d u$ choc:

$$
X^{\beta} V_{\beta}=W^{\beta} V_{\beta}=\operatorname{ar}\left(h^{\alpha} l_{\alpha}\right)(\alpha+\mu H) .
$$

D'après la définition de $\alpha$, on obtient le scalaire invariant:

$$
b=f\left(h^{\alpha} l_{\alpha}^{\prime}\right), \quad\left(X^{\beta} V_{\beta}=c^{2} a b\right) .
$$

Considérons enfin le scalaire invariant

Un calcul élémentaire [6] donne

$$
K=\frac{1}{c^{4} a^{2}} X^{\beta} X_{\beta}
$$

où l'on a posé:

$$
K=f^{2}-\frac{a^{2}}{l^{\alpha} l_{\alpha}} \tau^{2}+2 \mu \frac{\chi}{c^{2}} \tau-\frac{\mu^{2} H}{c^{4}} \chi
$$

$$
\chi=|h|^{2}+\frac{a^{2}}{l^{\alpha} l_{\alpha}} H \text {. }
$$

En évaluant $H K$, il vient:

$$
H K=\frac{b^{2}}{a^{2}}-\frac{1}{c^{4}} \chi \alpha^{2} .
$$

Ainsi $L=\chi \alpha^{2}$ est un invariant qui, $a, H, K$ étant fixés, peut être substitué à $b$, en convenant que le signe de $\left(h^{\alpha} l_{\alpha}\right)$ reste inchangé.

d) De cette étude, il résulte que les deux variables thermodynamiques du fluide et les trois scalaires $|h|^{2}, u^{\alpha} l_{\alpha}, h^{\alpha} l_{\alpha}$ vérifient les cinq relations:

$$
\begin{aligned}
r_{1} u_{1}^{\alpha} l_{\alpha} & =r_{0} u_{0}^{\alpha} l_{\alpha}=a, \\
\frac{\left(h_{1}^{\alpha} l_{\alpha}\right)^{2}}{a^{2}}-\frac{\left|h_{1}\right|^{2}}{r_{1}^{2}} & =\frac{\left(h_{0}^{\alpha} l_{\alpha}\right)^{2}}{a^{2}}-\frac{\left|h_{0}\right|^{2}}{r_{0}^{2}}=H, \\
q_{1}-\frac{c^{2} a^{2}}{l^{\alpha} l_{\alpha}} \tau_{1} & =q_{0}-\frac{c^{2} a^{2}}{l^{\alpha} l_{\alpha}} \tau_{0}=e, \\
f_{1}^{2}-\frac{a^{2}}{l^{\alpha} l_{\alpha}} \tau_{1}^{2}+2 \mu \frac{\chi_{1}}{c^{2}} \tau_{1}-\frac{\mu^{2} H}{c^{4}} \chi_{1} & =f_{0}^{2}-\frac{a^{2}}{l^{\alpha} l_{\alpha}} \tau_{0}^{2}+2 \mu \frac{\chi_{0}}{c^{2}} \tau_{0}-\frac{\mu^{2} H}{c^{4}} \chi_{0}=K, \\
\chi_{1} \alpha_{1}^{2} & =\chi_{0} \alpha_{0}^{2}=L .
\end{aligned}
$$


On peut considérer (10.12), (10.13), (10.14) comme définissant les valeurs de $\chi$ et des variables thermodynamiques après le choc; $(10.10)$ fournit alors la composante normale de la vitesse et (10.11) celle du champ magnétique.

D'autre part les composantes tangentielles de la vitesse et du champ magnétique vérifient, d'après l'invariance de $V^{\beta}$ et $W^{\beta}$ :

$$
\left.\begin{array}{r}
\left(h_{1}^{\alpha} l_{\alpha}\right) v_{1}^{\beta}-\left(u_{1}^{\alpha} l_{\alpha}\right) t_{1}^{\beta}=\left(h_{0}^{\alpha} l_{\alpha}\right) v_{0}^{\beta}-\left(u_{0}^{\alpha} l_{\alpha}\right) t_{0}^{\beta} \\
\left(c^{2} r_{1} f_{1}+\mu\left|h_{1}\right|^{2}\right)\left(u_{1}^{\alpha} l_{\alpha}\right) v_{1}^{\beta}-\mu\left(h_{1}^{\alpha} l_{\alpha}\right) t_{1}^{\beta} \\
=\left(c^{2} r_{0} f_{0}+\mu\left|h_{0}\right|^{2}\right)\left(u_{0}^{\alpha} l_{\alpha}\right) v_{0}^{\beta}-\mu\left(h_{0}^{\alpha} l_{\alpha}\right) t_{0}^{\beta}
\end{array}\right\}
$$

Le déterminant des premiers membres de (10.15) aux inconnues $v_{1}^{\beta}, t_{1}^{\beta}$ s'écrit:

$$
D_{1}(l)=\left(c^{2} r_{1} f_{1}+\mu\left|h_{1}\right|^{2}\right)\left(u_{1}^{\alpha} l_{\alpha}\right)^{2}-\mu\left(h_{1}^{\alpha} l_{\alpha}\right)^{2}=a^{2} \alpha_{1} .
$$

Si $\alpha_{1} \neq 0,(10.15)$ détermine $v_{1}^{\beta}, t_{1}^{\beta}$ en fonction de quantités connues d'après les équations scalaires.

\section{Cas singuliers}

a) Supposons $\alpha_{1}=0$ en un point $x$ de $\Sigma ; \Sigma$ est alors onde d'Alfven en $x$ pour l'état postérieur au choc et elle est orientée dans le temps $\left(l^{\alpha} l_{\alpha}<0\right)$. La relation $(10.14)$ donne

$$
\chi_{0} \alpha_{0}^{2}=0
$$

et ou bien $\alpha_{0}=0$, ou bien $\chi_{0}=0$.

Dans le cas $\alpha_{0}=\alpha_{1}=0, \Sigma$ définit un choc dit d'Alfven. Les cas $\alpha_{0} \neq 0$, $\chi_{0}=0, \alpha_{1}=0$ et $\alpha_{0}=0, \chi_{1}=0\left(\alpha_{1} \neq 0\right)$ sont dits des chocs singuliers.

Un choc d'Alfven peut être de type A ou B. Sous l'hypothèse $\tau_{p}^{\prime}<0$, j'ai établi ailleurs [5] que les variables thermodynamiques et les composantes normales de la vitesse et du champ magnétique sont invariantes au cours d'un choc d'Alfven. Les composantes tangentielles, de grandeurs invariantes, peuvent tourner au cours du choc en respectant la condition suivante: le vecteur $A^{\alpha}$ (resp. $B^{\alpha}$ ) reste invariant dans un choc d'Alfven de type $A$ (resp. $B$ ).

b) En ce qui concerne $\chi$, nous allons établir le lemme suivant qui nous sera utile à différentes reprises.

Lemme 1. Pour $l^{\alpha} l_{\alpha} \neq 0$, la quantité $\chi$ définie par (10.9) a le signe de $-\left(l^{\alpha} l_{\alpha}\right)$; pour que $\chi=0$ il faut et il suffit que $l$ appartienne au 2-plan $(u, h)$.

2. Si $l^{\alpha} l_{\alpha}$ est $\geqq 0$, on $a H \leqq 0$ et par suite $\alpha>0$.

En effet d'après (10.9) et la définition de $H$ :

$$
\left(l^{\alpha} l_{\alpha}\right) \chi=|h|^{2} l^{\alpha} l_{\alpha}+\left(h^{\alpha} l_{\alpha}\right)^{2}-|h|^{2}\left(u^{\alpha} l_{\alpha}\right)^{2}
$$


soit en introduisant $h_{n}^{2}$ :

$$
\left(l^{\alpha} l_{\alpha}\right) \chi=\left(h_{n}^{2}-|h|^{2}\right)\left(\left(u^{\alpha} l_{\alpha}\right)^{2}-l^{\alpha} l_{\alpha}\right)
$$

ce qui, compte-tenu du lemme du $\S 9$, démontre le $1^{\circ}$.

L'inégalité $h_{n}^{2} \leqq|h|^{2}$ peut s'écrire:

$$
\left(h^{\alpha} l_{\alpha}\right)^{2} \leqq|h|^{2}\left(\left(u^{\alpha} l_{\alpha}\right)^{2}-l^{\alpha} l_{\alpha}\right) .
$$

En divisant par $a^{2}=\left(r u^{\alpha} l_{\alpha}\right)^{2}$, il vient:

$$
H=\frac{\left(h^{\alpha} l_{\alpha}\right)^{2}}{a^{2}}-\frac{|h|^{2}}{r^{2}} \leqq-\frac{|h|^{2}}{a^{2}} l^{\alpha} l_{\alpha} .
$$

Pour $l^{\alpha} l_{\alpha} \geqq 0$, on a donc $H \leqq 0$ et $\alpha>0$.

12. Les vecteurs $U^{\beta}$ et $T^{\beta}$ pour un choc

a) Pour un état $Y$ du fluide en $x$, considérons le vecteur

$$
U^{\beta}=\frac{h^{\alpha} l_{\alpha}}{a} t^{\beta}-\frac{|h|^{2}}{r} v^{\beta} .
$$

Ce vecteur est orthogonal $\grave{a} \gamma^{\beta}$. En effet:

Or l'on a:

$$
U^{\beta} V_{\beta}=\frac{h^{\alpha} l_{\alpha}}{a} t^{\beta} V_{\beta}-\frac{|h|^{2}}{r} v^{\beta} V_{\beta} .
$$

$$
t^{\beta} V_{\beta}=h^{\beta} V_{\beta}=\frac{a}{r}|h|^{2}, \quad v^{\beta} V_{\beta}=h^{\beta} l_{\alpha} .
$$

Il vient ainsi:

$$
U^{\beta} V_{\beta}=\frac{|h|^{2}}{r}\left(h^{\alpha} l_{\alpha}\right)-\frac{|h|^{2}}{r}\left(h^{\alpha} l_{\alpha}\right)=0 .
$$

Transformons l'expression de $U^{\beta}$ en substituant à $t^{\beta}$ son expression en fonction de $V^{\beta}$. On a:

Par produit par $\alpha$, il vient:

$$
U^{\beta}=H r v^{\beta}-\frac{r h^{\alpha} l_{\alpha}}{a^{2}} V^{\beta}
$$

$$
\alpha U^{\beta}=\frac{H}{a}\left(a \alpha r v^{\beta}+\mu \frac{r h^{\alpha} l_{\alpha}}{a} V^{\beta}\right)-c^{2} \frac{b}{a^{2}} V^{\beta}
$$

c'est-à-dire:

$$
\alpha U^{\beta}=\frac{H}{a} X^{\beta}-c^{2} \frac{b}{a^{2}} V^{\beta} .
$$

Ainsi le vecteur $\alpha U^{\beta}$ est invariant au cours d'un choc défini par $\Sigma$.

Un calcul aisé à partir de (12.2) fournit le carré de $U^{\beta}$ :

$$
U^{\beta} U_{B}=-H \chi \text {. }
$$

b) Pour un état $Y$ du fluide posons $H=\varepsilon(Y)|H|$, où $H$ est $\neq 0$ (le cas $H=0$ pouvant être traité à part). Nous envisageons un choc non d'Alfven de telle sorte que $\alpha_{0}$ et $\alpha_{1}$ ne s'annulent pas simultanément. $\mathrm{Au}$ vecteur $U^{\beta}$, nous substituons le vecteur:

$$
T^{\beta}=\frac{1}{\sqrt{|H|}} U^{\beta}=\frac{h^{\alpha} l_{\alpha}}{a \sqrt{|H|}} t^{\beta}-\frac{|h|^{2}}{r \sqrt{|H|}} v^{\beta} .
$$


Ce vecteur est tel que $\alpha T^{\beta}$ est invariant au cours du choc et vérifie

$$
T^{\beta} T_{\beta}=-\varepsilon(Y) \chi .
$$

Pour les états $Y_{0}$ et $Y_{1}$, on a:

On en déduit:

$$
T_{1}^{\beta}=\frac{\alpha_{0}}{\alpha_{1}} T_{0}^{\beta} .
$$

Par suite:

$$
\left[T^{\beta}\right]=\left(\frac{\alpha_{0}}{\alpha_{1}}-1\right) T_{0}^{\beta} .
$$

$$
\left[T^{\beta}\right]\left[T_{\beta}\right]=\left(\frac{\alpha_{0}}{\alpha_{1}}-1\right)^{2} T_{0}^{\beta} T_{0 \beta}=-\varepsilon \chi_{0}\left(\frac{\alpha_{0}^{2}}{\alpha_{1}^{2}}+1-2 \frac{\alpha_{0}}{\alpha_{1}}\right)
$$

soit en tenant compte de (10.14):

$$
\left[T^{\beta}\right]\left[T_{\beta}\right]=-\varepsilon\left(\chi_{1}+\chi_{0}-2 \chi_{0} \frac{\alpha_{0}}{\alpha_{1}}\right) .
$$

Or, toujours d'après (10.14):

On obtient ainsi:

$$
\chi_{0} \frac{\alpha_{0}}{\alpha_{1}}=\chi_{1} \frac{\alpha_{1}}{\alpha_{0}}=\varepsilon^{\prime} \sqrt{\chi_{0} \bar{\chi}_{1}} .
$$

$$
\left[T^{\beta}\right]\left[T_{\beta}\right]=-\varepsilon\left(\chi_{1}+\chi_{0}-2 \varepsilon^{\prime} \sqrt{\chi_{0} \chi_{1}}\right), \quad\left(\varepsilon=\varepsilon\left(Y_{0}\right)=\varepsilon\left(Y_{1}\right)\right)
$$

où $\varepsilon^{\prime}= \pm 1$ a le signe de $L \alpha_{0} \alpha_{1}$.

\section{Fonction d'Hugoniot et orientation des ondes de choc}

\section{Fonction d'Hugoniot}

a) La relation (10.13) peut s'écrire:

$$
c^{2}\left[f^{2}\right]-\frac{c^{2} a^{2}}{l^{\alpha} l_{\alpha}}\left[\tau^{2}\right]+2 \mu[\chi \tau]-\frac{\mu^{2} H}{c^{2}}[\chi]=0 .
$$

En tirant $c^{2} a^{2} / l^{\alpha} l_{\alpha}$ de (10.12), il vient:

$$
c^{2}\left[f^{2}\right]-\left(\tau_{0}+\tau_{1}\right)[q]+2 \mu[\chi \tau]-\frac{\mu^{2} H}{c^{2}}[\chi]=0 .
$$

Nous substituerons désormais à (10.13) une relation qui, compte-tenu de (10.12) et (10.14) lui est équivalente. Un calcul aisé, mais un long (voir [5], p. 57-59) permet de déduire de (13.1) en faisant usage de (10.14), la relation dite d'Hugoniot

$$
c^{2}\left[f^{2}\right]-\left(\tau_{0}+\tau_{1}\right)[p]+[\tau] \frac{1}{2} \mu\left(\chi_{0}+\chi_{1}-2 \varepsilon^{\prime} \sqrt{\chi_{0} \chi_{1}}\right)=0 .
$$

D'après (12.6) cette relation peut s'écrire:

$$
c^{2}\left[f^{2}\right]-\left(\tau_{0}+\tau_{1}\right)[p]-[\tau] \frac{1}{2} \mu \varepsilon\left[T^{\beta}\right]\left[T_{\beta}\right]=0 .
$$

C'est (13.3) que nous substituons à $(10.13)^{2}$.

${ }^{2}$ Nous avais introduit ailleurs [9] une autre fonction d'Hugoniot, plus commode pour certains problèmes. 
b) Dans un état $Y$ du fluide distinguons un état partiel $Z$ défini par $\left(p, \tau, T^{\beta}\right)$. En nous limitant aux états $Y$ tels que $H(Y) H\left(Y_{0}\right)>0$, nous sommes conduits à introduire la fonction d'Hugoniot $\mathscr{H}$ de la magnétohydrodynamique considérée comme une fonction d'un état partiel $Z$ pour un état partiel initial fixé $Z_{0}$ :

$$
\begin{aligned}
\mathscr{H}\left(Z_{0}, Z\right)= & c^{2}\left(f^{2}-f_{0}^{2}\right)-\left(\tau+\tau_{0}\right)\left(p-p_{0}\right)-\left(\tau-\tau_{0}\right) \\
& \cdot \frac{1}{2} \mu \varepsilon\left(T^{\beta}-T_{0}^{\beta}\right)\left(T_{\beta}-T_{0 \beta}\right) .
\end{aligned}
$$

On a manifestement $\mathscr{H}\left(Z_{0}, Z_{0}\right)=0$ et (13.3) peut s'écrire:

$$
\mathscr{H}\left(Z_{0}, Z_{1}\right)=0 .
$$

c) Substituons, d'autre part, à $q$ la variable:

$$
\bar{q}=p+\frac{1}{2} \mu \chi=q+\frac{1}{2} \mu \frac{a^{2}}{l^{\alpha} l_{\alpha}} H .
$$

La relation (10.12) peut s'écrire sous la forme:

$$
\bar{q}_{1}-\bar{q}_{0}=\frac{c^{2} a^{2}}{l^{\alpha} l_{\alpha}}\left(\tau_{1}-\tau_{0}\right)
$$

chaque état partiel $Z=\left\{p, \tau, T^{\beta}\right\}$ détermine d'après (12.5) un point dans le plan $(\bar{q}, \tau)$.

d) Un état $Y_{0}$ étant fixé, considérons les états $Y$ du fluide vérifiant les conditions suivantes:

$$
\begin{aligned}
\left(\mathrm{C}_{1}\right): a^{2} H & =a_{0}^{2} H_{0}, \\
\left(\mathrm{C}_{2}\right): \bar{q}-\bar{q}_{0} & =\frac{c^{2} a^{2}}{l^{\alpha} l_{\alpha}}\left(\tau-\tau_{0}\right), \\
\left(\mathrm{C}_{3}\right): c^{2}\left(\tau T^{\beta}-\tau_{0} T_{0}^{\beta}\right) & =\mu H\left(T^{\beta}-T_{0}^{\beta}\right)
\end{aligned}
$$

où les grandeurs munies de l'indice 0 correspondent à l'état $Y_{0}$ et celles sans indice 0 à l'état $Y$. Il est clair que l'état $Y_{1}$ satisfait ces conditions.

Par ces conditions, $Y$ est soumis à cinq relations scalaires et dépend encore de trois paramètres. L'un peut être pris égal à $a$, le second fixant la position du point représentatif dans le plan $(\bar{q}, \tau)$ de la droite définie par $\left(\mathrm{C}_{2}\right)$ et le troisième achevant [compte-tenu de $\left(\mathrm{C}_{3}\right)$ ] de fixer les composantes tangentielles $v^{\beta}$ et $t^{\beta}$.

Nous nous proposons d'évaluer la différentielle de la fonction d'Hugoniot $\mathscr{H}$ dans la famille définie par les conditions précédentes.

\section{Différentielle de $\mathscr{H}$}

Pour abréger les notations, nous allons introduire dans ce paragraphe le crochet pour représenter les différences de quantités correspondant respectivement à l'état $Y$ et à l'état $Y_{0}$. Les conditions précédentes 
s'écrivent

$$
\begin{aligned}
\left(\mathrm{C}_{1}\right): & {\left[\alpha^{2} H\right] } & =0, \\
\left(\mathrm{C}_{2}\right): & {[\bar{q}] } & =\frac{c^{2} a^{2}}{l^{\alpha} l_{\alpha}}[\tau], \\
\left(\mathrm{C}_{3}\right): & c^{2}\left[\tau T^{\beta}\right] & =\mu H\left[T^{\beta}\right] .
\end{aligned}
$$

a) Commençons par différentier $\left(\mathrm{C}_{2}\right)$. $\mathrm{Il}$ vient:

$$
d \bar{q}=\frac{c^{2}}{l^{\alpha} l_{\alpha}}\left([\tau] d a^{2}+a^{2} d \tau\right) .
$$

Par produit par $[\tau]$ et compte-tenu de $\left(\mathrm{C}_{2}\right)$, on obtient:

$$
[\tau] d \bar{q}-[\bar{q}] d \tau=\frac{c^{2}}{l^{\alpha} l_{\alpha}}[\tau]^{2} d a^{2} .
$$

En substituant à $\bar{q}$ sa valeur, il vient la relation:

$$
[\tau] d p-[p] d \tau=-\frac{1}{2} \mu[\tau] d \chi+\frac{1}{2} \mu[\chi] d \tau+\frac{c^{2}}{l^{\alpha} l_{\alpha}}[\tau]^{2} d a^{2} .
$$

Différentions de même $\left(\mathrm{C}_{3}\right)$ :

$$
c^{2} d\left(\tau T^{\beta}\right)=\mu H d T^{\beta}+\mu\left[T^{\beta}\right] d H .
$$

En multipliant par $\left[T_{\beta}\right]$, on obtient:

$$
c^{2}\left[T_{\beta}\right]\left(T^{\beta} d \tau+\tau d T^{\beta}\right)=\mu H\left[T_{\beta}\right] d T^{\beta}+\mu\left[T_{\beta}\right]\left[T^{\beta}\right] d H .
$$

Soit, compte-tenu de $\left(\mathrm{C}_{3}\right)$,

$$
\left(\left[\tau T_{\beta}\right]-\tau\left[T_{\beta}\right]\right) d T^{\beta}=\left[T_{\beta}\right] T^{\beta} d \tau-\frac{\mu}{c^{2}}\left[T_{\beta}\right]\left[T^{\beta}\right] d H .
$$

Or:

$$
\left[\tau T_{\beta}\right]-\tau\left[T_{\beta}\right]=\tau T_{\beta}-\tau_{0} T_{0 \beta}-\tau T_{\beta}+\tau T_{0 \beta}=[\tau] T_{0 \beta}
$$

Il vient ainsi la relation

$$
[\tau] T_{0 \beta} d T^{\beta}=\left[\tau_{\beta}\right] T^{\beta} d \tau-\frac{\mu}{c^{2}}\left[T_{\beta}\right]\left[T^{\beta}\right] d H .
$$

b) Considérons maintenant la fonction:

$$
\mathscr{H}=c^{2}\left[f^{2}\right]-\left(\tau+\tau_{0}\right)[p]-[\tau] \frac{1}{2} \mu \varepsilon\left[T^{\beta}\right]\left[T_{\beta}\right] .
$$

En tenant compte de:

$$
c^{2} f d f=f \Theta d S+\tau d p
$$

on obtient en différentiant:

$$
\begin{gathered}
d \mathscr{H}=2 f \Theta d S+[\tau] d p-[p] d \tau-[\tau] \frac{1}{2} \mu \varepsilon d\left(\left[T^{\beta}\right]\left[T_{\beta}\right]\right) \\
-\frac{1}{2} \mu \varepsilon\left[T^{\beta}\right]\left[T_{\beta}\right] d \tau .
\end{gathered}
$$

On en déduit d'après (14.1):

$$
\begin{aligned}
d \mathscr{H}=2 f \Theta d S+\frac{c^{2}}{l^{\alpha} l_{\alpha}}[\tau]^{2} d a^{2} & -\frac{1}{2} \mu \varepsilon[\tau]\left\{d\left(\left[T^{\beta}\right]\left[T_{\beta}\right]\right)+\varepsilon d \chi\right\} \\
& -\frac{1}{2} \mu \varepsilon\left(\left[T^{\beta}\right]\left[T_{\beta}\right]-\varepsilon[\chi]\right) d \tau .
\end{aligned}
$$


Or on a, compte tenu de (12.5):

$$
\left[T^{\beta}\right]\left[T_{\beta}\right]=T^{\beta} T_{\beta}+T_{0 \beta} T_{0}^{\beta}-2 T_{0 \beta} T^{\beta}, \quad \varepsilon[\chi]=-T_{\beta} T^{\beta}+T_{0 \beta} T_{0}^{\beta}
$$

Il en résulte:

D'autre part:

$$
\left[T^{\beta}\right]\left[T_{\beta}\right]-\varepsilon[\chi]=2\left[T_{\beta}\right] T^{\beta}
$$

$$
d\left(\left[T^{\beta}\right]\left[T_{\beta}\right]\right)=2 T_{\beta} d T^{\beta}-2 T_{0 \beta} d T^{\beta}, \quad \varepsilon d \chi=-2 T_{\beta} T^{\beta} .
$$

Il vient:

$$
d\left(\left[T^{\beta}\right]\left[T_{\beta}\right]\right)+\varepsilon d \chi=-2 T_{0 \beta} d T^{\beta} .
$$

En reportant dans (14.3), on obtient:

$$
d \mathscr{H}=2 f \Theta d S+\frac{c^{2}}{l^{x} l_{\alpha}}[\tau]^{2} d a^{2}+\mu \varepsilon\left([\tau] T_{0 \beta} d T^{\beta}-\left[T_{\beta}\right] T^{\beta} d \tau\right)
$$

soit, d'après (14.2):

$$
d \mathscr{H}=2 f \Theta d S+\frac{c^{2}}{l^{\alpha} l_{\alpha}}[\tau]^{2} d \alpha^{2}-\frac{\mu^{2} \varepsilon}{c^{2}}\left[T_{\beta}\right]\left[T^{\beta}\right] d H .
$$

Ce qui peut s'écrire, compte-tenu de $\left(\mathrm{C}_{3}\right)$,

$$
d \mathscr{H}=2 f \Theta d S+\frac{c^{2}}{l^{\alpha} l_{\alpha}}[\tau]^{2} d a^{2}-\frac{c^{2}}{|H|}\left[\tau T_{\beta}\right]\left[\tau T^{\beta}\right] \frac{d H}{H} .
$$

Or on déduit de $\left(\mathrm{C}_{1}\right)$ :

$$
\frac{d H}{H}=-\frac{d a^{2}}{a^{2}} \text {. }
$$

Il vient ainsi:

$$
d \mathscr{H}=2 f \Theta d S+\left\{\frac{[\tau]^{2}}{l^{\alpha} l_{\alpha}}+\frac{\left[\tau T_{\beta}\right]\left[\tau T^{\beta}\right]}{a^{2}|H|}\right\} c^{2} d a^{2} .
$$

c) Par un raisonnement analogue à celui du $\S 12, \mathrm{~b}$, il est aisé d'étudier, sous les hypothèses faites, le signe de $\left[T_{\beta}\right]\left[T^{\beta}\right]$ qui est aussi le signe de $\left[\tau T_{\beta}\right]\left[\tau T^{\beta}\right]$. On a d'après $\left(\mathrm{C}_{3}\right)$ :

$$
c^{2}\left(\tau T^{\beta}-\tau_{0} T_{0}^{\beta}\right)=\mu H T^{\beta}-\mu H T_{0}^{\beta}
$$

ce qui peut s'écrire:

On en déduit:

$$
\alpha T^{\beta}=\left(\alpha_{0}-\mu[H]\right) T_{0}^{\beta}
$$

Il vient ainsi:

$$
\left[T^{\beta}\right]=\left(\frac{\alpha_{0}}{\alpha}-1-\frac{\mu|H|}{\alpha}\right) T_{0}^{\beta}
$$

$$
\left[T_{\beta}\right]\left[T^{\beta}\right]=-\varepsilon \chi_{0}\left(\frac{\alpha_{0}}{\alpha}-1-\frac{\mu[H]}{\alpha}\right)^{2} .
$$

Ainsi $\left[T_{\beta}\right]\left[T^{\beta}\right]$ a le signe de $-\varepsilon \chi_{0}$. Nous pouvons énoncer:

Théorème. En différentiant $\mathscr{H}\left(Z_{0}, Z\right)$ dans la famille définie par les conditions $\left(\mathrm{C}_{1}\right),\left(\mathrm{C}_{2}\right),\left(\mathrm{C}_{3}\right)$ on obtient:

$d \mathscr{H}=2 f \Theta d S+\left\{\frac{\left(\tau-\tau_{0}\right)^{2}}{l^{\alpha} l_{\alpha}}+\frac{\left(\tau T_{\beta}-\tau_{0} T_{0 \beta}\right)\left(\tau T^{\beta}-\tau_{0} T_{0}^{\beta}\right)}{a^{2}|H|}\right\} c^{2} d a^{2}$

ou $\varepsilon\left(\tau T_{\beta}-\tau_{0} T_{0 \beta}\right)\left(\tau T^{\beta}-\tau_{0} T_{0}^{\beta}\right)$ a le signe de $-\chi_{0}$. 
15. Différentielle de $S$ le long d'une droite du plan $(\bar{q}, \tau)$

a) Ajoutons aux conditions $\left(\mathrm{C}_{1}\right),\left(\mathrm{C}_{2}\right),\left(\mathrm{C}_{3}\right)$ la condition

$$
\left(\mathrm{C}_{4}\right): \quad a=a_{0} \text {. }
$$

Les conditions $\left(\mathrm{C}_{1}\right)$ et $\left(\mathrm{C}_{3}\right)$ peuvent alors s'écrire:

$$
\begin{aligned}
& \left(\mathrm{C}_{1}^{\prime}\right): \quad H=H_{0}, \\
& \left(\mathrm{C}_{3}^{\prime}\right): \alpha T^{\beta}=\alpha_{0} T_{0}^{\beta}
\end{aligned}
$$

et $\left(\mathrm{C}_{3}^{\prime}\right)$ implique $L(Y)=L\left(Y_{0}\right)$. D'après $\left(\mathrm{C}_{2}\right)$, le point correspondant à $Z$ dans le plan $(\bar{q}, \tau)$ décrit la droite d'équation

$$
\bar{q}-\bar{q}_{0}=\frac{c^{2} a^{2}}{l^{\alpha} l_{\alpha}}\left(\tau-\tau_{0}\right)
$$

où $a=a_{0}$ est une constante donnée.

b) Nous nous proposons de donner une expression de la différentielle de $S$ quand $Y$ varie dans la famille d'états vérifiant les conditions $\left(\mathrm{C}_{1}\right)$, $\left(\mathrm{C}_{2}\right),\left(\mathrm{C}_{3}\right),\left(\mathrm{C}_{4}\right)$. De

et de (4.4) on déduit:

$$
d \tau=\tau_{p}^{\prime} d p+\tau_{S}^{\prime} d S
$$

$$
\tau_{S}^{\prime} d S=\frac{\gamma-1}{c^{2} r^{2}} d p+d \tau .
$$

Or en différentiant $\left(\mathrm{C}_{2}\right)$ avec $a=$ const. on a :

$$
d p=\frac{c^{2} a^{2}}{l^{\alpha} l_{\alpha}} d \tau-\frac{1}{2} \mu d \chi
$$

et en différentiant $L=\chi \alpha^{2}=$ const.

$$
\alpha d \chi+2 \chi c^{2} d \tau=0 \text {. }
$$

On en déduit:

$$
\tau_{S}^{\prime} \alpha d S=\left\{\frac{\gamma-1}{r^{2}}\left(\frac{a^{2}}{l^{\alpha} l_{\alpha}} \alpha+\mu \chi\right)+\alpha\right\} d \tau
$$

ce qui peut s'écrire, compte-tenu des valeurs de $\alpha$ et $\chi$ :

$$
\tau_{S}^{\prime} \alpha d S=\left\{\frac{\gamma-1}{r^{2}}\left(\frac{a^{2}}{l^{\alpha} l_{\alpha}} c^{2} \tau+\mu|h|^{2}\right)+\alpha\right\} d \tau .
$$

c) Le second membre de (15.1) peut s'évaluer aisément en fonction de $P(l)$. La relation $(8-1)$ définissant $P(l)$ peut se mettre sous la forme:

$$
\frac{P(l)}{a^{2}}=c^{2} \tau \frac{a^{2}}{r^{2}}(\gamma-1)+\left(c^{2} \tau+\mu \frac{|h|^{2}}{r^{2}} \gamma\right) l^{\alpha} l_{\alpha}-\mu \frac{\left(h^{\alpha} l_{\alpha}\right)^{2}}{a^{2}} l^{\alpha} l_{\alpha}
$$

ce qui peut s'écrire:

$$
\frac{P(l)}{a^{2}}=\frac{\gamma-1}{r^{2}}\left(c^{2} a^{2} \tau+\mu|h|^{2} l^{\alpha} l_{\alpha}\right)+\left(c^{2} \tau-\mu \frac{\left(h^{\alpha} l_{\alpha}\right)^{2}}{a^{2}}+\mu \frac{|h|^{2}}{r^{2}}\right) l^{\alpha} l_{\alpha} .
$$

Il en résulte:

$$
\frac{P(l)}{a^{2}}=\frac{\gamma-1}{r^{2}}\left(c^{2} a^{2} \tau+\mu|h|^{2} l^{\alpha} l_{\alpha}\right)+\alpha l^{\alpha} l_{\alpha} .
$$


On en déduit ainsi de (15.1) la relation inportante:

$$
\tau_{S}^{\prime} \alpha d S=\frac{P(l)}{a^{2} l^{\alpha} l_{\alpha}} d \tau
$$

16. Orientation dans le temps des ondes de choc

a) Considérons au point $x \in \Sigma$ un choc qui n'est ni nul, ni choc d'Alfven. On sait [5] que s'il en est ainsi, on a $\alpha_{1} \neq \alpha_{0}$ donc $\tau_{1} \neq \tau_{0}$. Nous nous proposons de démontrer que sous les hypothèses $\left(H_{1}\right)$ de compressibilité $\tau_{p}^{\prime}<0, \tau_{S}^{\prime}>0$ que nous postulons, l'onde de choc magnétohydrodynamique $\Sigma$ est orientée dans le temps.

Considérons la famille des états $Y$ vérifiant $\left(\mathrm{C}_{1}\right),\left(\mathrm{C}_{2}\right),\left(\mathrm{C}_{3}\right),\left(\mathrm{C}_{4}\right)$. Les points correspondants du plan $(\bar{q}, \tau)$ varient sur la droite portant le segment $\left(\bar{q}_{0}, \tau_{0}\right),\left(\bar{q}_{1}, \tau_{1}\right)$ que nous notons $\left(Z_{0}, Z_{1}\right)$ par abus de notation.

Il résulte de (14.6) et de (15.2) que l'on a dans ces conditions:

$$
d \mathscr{H}=2 f \Theta d S
$$

et

$$
\tau_{S}^{\prime} \alpha d S=\frac{P(l)}{a^{2} l^{\alpha} l_{\alpha}} d \tau
$$

b) Si $l^{\alpha} l_{\alpha}>0, y^{\Sigma}$ est $>1$ et on déduit de (9.4), soit:

$$
\frac{\left(1-y^{\Sigma}\right)^{2} P(l)}{\left(l^{\beta} l_{\beta}\right)^{2}}=\Pi\left(y^{\Sigma}\right)
$$

que $P(l)$ est $>0$. On sait d'après le lemme du $\S 11$ que $\alpha$ est $>0$. Ainsi, sous les hypothèses $\left(H_{1}\right)$, il résulte de (16.2) que $d S / d \tau$ est strictement positif le long de la droite $\left(Z_{0}, Z_{1}\right)$. Comme

$$
\mathscr{H}\left(Z_{0}, Z_{0}\right)=\mathscr{H}\left(Z_{0}, Z_{1}\right)=0
$$

la fonction $\mathscr{H}$ considérée comme dépendant de $\tau$ est stationnaire en un point au moins du segment $\left(Z_{0}, Z_{1}\right)$ et d'après $(16.1)$, il en est de même pour $S(\tau)$, ce qui est en contradiction avec $d S / d \tau>0$.

Si $l^{\alpha} l_{\alpha}=0, P(l)=c^{2} r f(\gamma-1)\left(u^{\alpha} l_{\alpha}\right)^{4}$ est $>0$ et $\alpha$ est toujours $>0$. On peut paramétriser le segment $\left(Z_{0}, Z_{1}\right)$ à l'aide de la variable $\bar{q}$ et substituer à (16.2):

$$
\tau_{S}^{\prime} \propto d S=\frac{P(l)}{c^{2} a^{4}} d \bar{q}
$$

Le même raisonnement appliqué à $S$ et $\mathscr{H}$ considérés comme fonctions de $\bar{q}$ conduit à la même contradiction.

Ainsi nécessairement $l^{\alpha} l_{\alpha}$ est $<0$ et $\Sigma$ est orientée dans le temps. Nous énonçons:

Théorème. Sous les hypothèses $\left(H_{1}\right)\left(\tau_{p}^{\prime}<0, \tau_{S}^{\prime}>0\right)$ toute onde de choc magnétohydrodynamique $\Sigma$ est nécessairement orientée dans le temps. Si $v_{0}^{\Sigma}$ et $v_{1}^{\Sigma}$ sont les vitesses de $\Sigma$ par rapport au fluide avant et apres le choc, on $a v_{0}^{\Sigma}<c, v_{1}^{\Sigma}<c$. 
En vertu du lemme du $\S 11$, nous pouvons poser $\chi=k^{2} \geqq 0$. D'après (13.2) la relation d'Hugoniot peut s'écrire ${ }^{3}$ :

$$
\begin{gathered}
\mathscr{H}\left(Z_{0}, Z_{1}\right)=c^{2}\left(f_{1}^{2}-f_{0}^{2}\right)-\left(\tau_{0}+\tau_{1}\right)\left(p_{1}-p_{0}\right)+\left(\tau_{1}-\tau_{0}\right) \\
\cdot \frac{1}{2} \mu\left(k_{1}-k_{0}\right)^{2}=0 .
\end{gathered}
$$

c) Dans la formule (14.6) donnant la différentielle de $\mathscr{H}$ sous les conditions $\left(\mathrm{C}_{1}\right),\left(\mathrm{C}_{2}\right),\left(\mathrm{C}_{3}\right)$ le coefficient de da $a^{2}$ est négatif, ce qui étend un résultat de la magnétohydrodynamique classique.

\section{Thermodynamique des chocs et vitesses des ondes de choc}

17. Thermodynamique des chocs

a) $p$ et $S$ étant les variables thermodynamiques de base, $f(p, S)$ vérifie d'après (3.4):

$$
c^{2} f_{p}^{\prime}=V>0, \quad c^{2} f_{S}^{\prime}=\Theta>0 .
$$

On en déduit par dérivation:

$$
\frac{\partial}{\partial p}\left(c^{2} f^{2}\right)=2 \tau
$$

Les états $Z_{0}$ et $Z_{1}$ sont reliés par la relation d'Hugoniot (16.3) qui est symétrique par rapport aux deux états.

Au cours d'un choc, on a nécessairement en chaque point de $\Sigma$

$$
S_{0} \leqq S_{1} .
$$

Nous allons établir le résultat suivant, valable en chaque point de $\Sigma$

Théorème 1. Pour un choc qui n'est ni nul, ni d'Alfven, on a sous les hypothèses de compressibilité $\left(H_{1}\right),\left(H_{2}\right)$ :

$$
S_{0}<S_{1} \text {. }
$$

En effet supposons qu'au point $x$ de $\Sigma$, on ait $S_{0}=S_{1}$ et $p_{0} \neq p_{1}$. En modifiant au besoin le numérotage des états $Z_{0}$ et $Z_{1}$, on peut supposer $p_{0}<p_{1}$. On a alors $\tau_{0}>\tau_{1}$ puisque $\tau_{p}^{\prime}<0$. De (17.2), on déduit:

$$
c^{2}\left\{f^{2}\left(p_{1}, S_{0}\right)-f^{2}\left(p_{0}, S_{0}\right)\right\}=2 \int_{p_{0}}^{p_{1}} \tau\left(p, S_{0}\right) d p .
$$

Il en résulte d'après l'hypothèse de convexité $\left(H_{2}\right)$ :

soit

$$
c^{2}\left(f_{1}^{2}-f_{0}^{2}\right)<\left(p_{1}-p_{0}\right)\left(\tau\left(p_{0}, S_{0}\right)+\tau\left(p_{1}, S_{0}\right)\right)
$$

$$
c^{2}\left(f_{1}^{2}-f_{0}^{2}\right)-\left(\tau_{1}+\tau_{0}\right)\left(p_{1}-p_{0}\right)<0 .
$$

${ }^{3}$ Sous les conditions $H=H_{0}$ et $\alpha T_{\beta}=\alpha_{0} T_{0 \beta}, \mathscr{H}$ coincide avec $\mathscr{H}^{*}$ donné par:

$$
\mathscr{H}^{*}\left(p, \tau ; p_{0} \tau_{0}\right)=c^{2}\left(f^{2}-f_{0}^{2}\right)-\left(\tau+\tau_{0}\right)\left(p-p_{0}\right)+\left(\tau-\tau_{0}\right) \frac{1}{2} \mu\left(k-k_{0}\right)^{2}
$$

ou $H=H_{0}, k\left(c^{2} \tau-\mu H\right)=L_{0}$. 
On déduit de la relation d'Hugoniot que $\tau_{1}>\tau_{0}$, ce qui implique contradiction. On a donc $p_{0}=p_{1}$ et le choc envisagé ne peut être que nul ou d'Alfven.

b) Cela posé, nous nous proposons de démontrer le théorème suivant.

Théorème 2. Sous les hypothèses de compressibilité $\left(H_{1}\right),\left(H_{2}\right)$, on a pour un choc qui n'est ni nul ni choc d'Alfven

$$
p_{1}>p_{0}, \quad f_{1}>f_{0}, \quad \tau_{1}<\tau_{0} .
$$

En particulier toute onde de choc est une onde de compression et $V_{1}<V_{0}$.

Supposons en effet $p_{1} \leqq p_{0}$ au point $x$ de $\Sigma$. De (17.2) on déduit

$$
c^{2}\left\{f^{2}\left(p_{0}, S_{0}\right)-f^{2}\left(p_{1}, S_{0}\right)\right\}=2 \int_{p_{1}}^{p_{0}} \tau\left(p, S_{0}\right) d p .
$$

D'après la condition de convexité $\tau_{p^{2}}^{\prime \prime}>0$, il en résulterait

$$
c^{2}\left\{f^{2}\left(p_{0}, S_{0}\right)-f^{2}\left(p_{1}, S_{0}\right)\right\} \leqq\left(p_{0}-p_{1}\right)\left(\tau\left(p_{0}, S_{0}\right)+\tau\left(p_{1}, S_{0}\right)\right) .
$$

Comme $S_{0}<S_{1}$ on aurait a fortiori, puisque $f_{S}^{\prime}>0, \tau_{S}^{\prime}>0$

soit

$$
c^{2}\left\{f^{2}\left(p_{0}, S_{0}\right)-f^{2}\left(p_{1}, S_{1}\right)\right\}<\left(p_{0}-p_{1}\right)\left(\tau\left(p_{0}, S_{0}\right)+\tau\left(p_{1}, S_{1}\right)\right)
$$

$$
c^{2}\left(f_{1}^{2}-f_{0}^{2}\right)-\left(\tau_{1}+\tau_{0}\right)\left(p_{1}-p_{0}\right)>0 .
$$

La relation d'Hugoniot donne alors $\tau_{1}<\tau_{0}$. D'après $\left(H_{1}\right)$ cela est contradictoire avec $p_{1} \leqq p_{0}, S_{1}>S_{0}$. On a donc $p_{1}>p_{0}$ et d'après (17.1) $f_{1}>f_{0}$.

Pour établir $\tau_{1}<\tau_{0}$ on part de:

$$
c^{2}\left\{f^{2}\left(p_{1}, S_{1}\right)-f^{2}\left(p_{0}, S_{1}\right)\right\}=2 \int_{p_{0}}^{p_{1}} \tau\left(p, S_{1}\right) d p .
$$

Il en résulte puisque $\tau_{p}^{\prime}<0$

$$
c^{2}\left\{f^{2}\left(p_{1}, S_{1}\right)-f^{2}\left(p_{0}, S_{1}\right)\right\}>2 \tau\left(p_{1}, S_{1}\right)\left(p_{1}-p_{0}\right)
$$

ou, a fortiori,

$$
c^{2}\left(f_{1}^{2}-f_{0}^{2}\right)-2 \tau_{1}\left(p_{1}-p_{0}\right)>0 .
$$

De la relation d'Hugoniot il résulte alors :

$$
\left(\tau_{1}-\tau_{0}\right)\left\{p_{1}-p_{0}+\frac{1}{2} \mu\left(k_{1}-k_{0}\right)^{2}\right\}<0
$$

soit $\tau_{1}<\tau_{0}$ ce qui démontre le théorème.

On a par suite $\alpha_{1}<\alpha_{0}$.

\section{Onde de choc et ondes d'Alfven}

Considérons à la traversée de $\Sigma$ un choc non tangentiel qui ne soit pas choc d'Alfven.

a) Nous nous proposons d'établir le lemme suivant. 
Lemme. Il existe toujours une direction $m$ au moins orthogonale $\grave{a} l$, $u_{0}, u_{1}, h_{0}, h_{1}$.

Examinons les différents cas possibles.

1. Si $\alpha_{0} \alpha_{1} \neq 0$, il existe une direction au moins orthogonale aux vecteurs $l, V$ et $X$ avec:

$$
X^{\beta}=a \alpha_{0} r_{0} v_{0}^{\beta}+\mu \frac{r_{0}}{a} h_{0}^{\alpha} l_{\alpha} V^{\beta}=a \alpha_{1} r_{1} v_{1}^{\beta}+\mu \frac{r_{1}}{a} h_{1}^{\alpha} l_{\alpha} V^{\beta} .
$$

De (18.1) il résulte que $m$ est orthogonal à $v_{0}$ et $v_{1}$ donc à $u_{0}$ et $u_{1}$. Comme

$$
V^{\beta}=\left(h_{0}^{\alpha} l_{\alpha}\right) u_{0}^{\beta}-\frac{a}{r_{0}} h_{0}^{\beta}=\left(h_{1}^{\alpha} l_{\alpha}\right) u_{1}^{\beta}-\frac{a}{r_{1}} h_{1}^{\beta}
$$

$m$ est aussi orthogonale à $h_{0}$ et à $h_{1}$.

2. Si $\alpha_{0} \neq 0, \alpha_{1}=0$, on a $\chi_{0}=0$ et il résulte du lemme du $\S 11$ que $l$ est dans le 2 -plan $\left(u_{0}, h_{0}\right)$. Le vecteur $V$ est orthogonale à $l$ dans ce 2 -plan.

Il existe une direction $m$ au moins orthogonale à $l, V$ et $u_{1}$. Cette direction est orthogonale au 2-plan $u_{0}, h_{0}$ et, étant orthogonale à $V$ et $u_{1}$ est orthogonale à $h_{1}$.

3. Si $\alpha_{0}=0, \alpha_{1} \neq 0$, il suffit d'échanger le rôle des indices 0 et 1 .

b) En $x \in \sum$ introduisons une perturbation infinitésimale de l'état antérieur au choc. Il en résulte une perturbation infinitésimale de l'état postérieur au choc reliée à la précédente par les relations obtenues en différentiant les équations fondamentales de choc. Adoptons en $x$ un repère orthonormé $\left\{e_{(\alpha)}\right\}$ tel que $e_{(1)}$ soit colinéaire à $l$ et $e_{(3)}$ à $m$. Dans ce repère, il vient:

$$
u_{0}^{3}=0, \quad h_{0}^{3}=0, \quad u_{1}^{3}=0, \quad h_{1}^{3}=0 .
$$

Le système différentiel se partage en deux systèmes dont le premier contient exclusivement les perturbations $\delta u^{3}, \delta h^{3}$, soit:

$$
\left.\begin{array}{rl}
\left(h_{1}^{\alpha} l_{\alpha}\right) \delta u_{1}^{3}-\frac{a}{r_{1}} \delta h_{1}^{3} & =\left(h_{0}^{\alpha} l_{\alpha}\right) \delta u_{0}^{3}-\frac{a}{r_{0}} \delta h_{0}^{3} \\
\beta_{1}^{2} \frac{a}{r_{1}} \delta u_{1}^{3}-\left(h_{1}^{\alpha} l_{\alpha}\right) \delta h_{1}^{3} & =\beta_{0}^{2} \frac{a}{r_{0}} \delta u_{0}^{3}-\left(h_{0}^{\alpha} l_{\alpha}\right) \delta h_{0}^{3} .
\end{array}\right\}
$$

Nous supposons que seuls $\delta u_{0}^{3}, \delta h_{0}^{3}$ sont $\neq 0$. Les variables thermodynamiques n'ayant pas été perturbées, il en résulte que, dans les états respectivement antérieur ou postérieur au choc $\Sigma$, de telles perturbations correspondent à des chocs d'Alfven infinitésimaux, c'est-à-dire à des ondes d'Alfven.

Considérons, dans l'état antérieur à $\Sigma$, une onde d'Alfven de type $A$. Le vecteur $A_{0}^{\alpha}$ étant invariant à la traversée de cette onde, une telle onde porte en $x$ une perturbation $\left(\delta u_{0 A}^{3}, \delta h_{0 A}^{2}\right)$ telle que

$$
\beta_{0} \delta u_{0 A}^{3}+\delta h_{0 A}^{3}=0 \text {. }
$$

De même une onde d'Alfven de type $B$ porte en $x$ une perturbation $\left(\delta u_{0 B}^{3}, \delta h_{0 B}^{3}\right)$ telle que:

$$
\beta_{0} \delta u_{0 B}^{3}-\delta h_{0 B}^{3}=0
$$


La superposition en $x$ d'une onde de type $A$ et d'une onde de type $B$ fournit une perturbation $\left(\delta u_{0}^{3}, \delta h_{0}^{3}\right)$ arbitraire.

c) Les vecteurs $A_{0}^{\alpha}$ et $B_{0}^{\alpha}$ vérifient en $x \in \Sigma$ :

On en déduit:

$$
A_{0}^{\alpha} l_{\alpha}=\beta_{0} \frac{a}{r_{0}}+h_{0}^{\alpha} l_{\alpha}, \quad B_{0}^{\alpha} l=\beta_{0} \frac{r_{0}}{a}-h_{0}^{\alpha} l_{\alpha} .
$$

$$
\left(A_{0}^{\alpha} l_{\alpha}\right)\left(B_{0}^{\alpha} l_{\alpha}\right)=\beta_{0}^{2} \frac{a^{2}}{r_{0}^{2}}-\left(h_{0}^{\alpha} l_{\alpha}\right)^{2}=\frac{a^{2}}{\mu} \alpha_{0} .
$$

Convenons d'orienter $l$ de l'état antérieur vers l'état postérieur au choc $\Sigma$; on a alors $a<0$. Supposons pour fixer les idées $b>0$ et par suite $h_{0}^{\alpha} l_{\alpha}$ (resp. $\left.h_{0}^{\alpha} l_{\alpha}\right)>0$. D'après (18.6), le vecteur $B_{0}$ (resp. $B_{1}$ ) est orienté par rapport à $\Sigma$ du même côté que $l$. Quant au vecteur $A_{0}$ (resp. $A_{1}$ ) son orientation par rapport à $\Sigma$ est celle de $l$ ou l'orientation opposée selon que $\alpha_{0}$ (resp. $\alpha_{1}$ ) est positif ou négatif. Pour $\alpha$ nul, $A$ est tangent à $\Sigma$. Si $b$ était supposé $<0$, les rôles des vecteurs $A$ et $B$ seraient simplement inversés.

19. Compatibilité d'une onde de choc avec les ondes d'Alfven

a) Nous examinons les cas où le choc $\Sigma$ envisagé, non choc d'Alfven, est tel que $\alpha_{0} \alpha_{1}=0$.

Supposons d'abord:

$$
\alpha_{1}=0, \quad \alpha_{0}>0 .
$$

Dans l'état $Y_{0}$, les ondes d'Alfven de type $A$ et $B$ qui aboutissent en $x \in \Sigma$ peuvent créer en ce point une perturbation $\left(\delta u_{0}^{3}, \delta h_{0}^{3}\right)$ arbitraire et dans les relations (18.3), on a puisque $\alpha_{1}=0$

$$
\beta_{1}^{2} \frac{a^{2}}{r_{1}^{2}}-\left(h_{1}^{\alpha} l_{\alpha}\right)^{2}=0 .
$$

Pour que ces relations admettent une solution quels que soient $\delta u_{0}^{3}, \delta h_{0}^{3}$, il faut et il suffit que l'on ait identiquement en $\delta u_{0}^{3}, \delta h_{0}^{3}$ :

soit:

$$
\beta_{1}^{2} \frac{a}{r_{1}}\left(h_{0}^{\alpha} l_{\alpha} \delta u_{0}^{3}-\frac{a}{r_{0}} \delta h_{0}^{3}\right)-h_{1}^{\alpha} l_{\alpha}\left(\beta_{0}^{2} \frac{a}{r_{0}} \delta u_{0}^{3}-h_{0}^{\alpha} l_{\alpha} \delta h_{0}^{3}\right)=0
$$

$$
\begin{aligned}
\beta_{1}^{2} \frac{a}{r_{1}}\left(h_{0}^{\alpha} l_{\alpha}\right)-\beta_{0}^{2} \frac{a}{r_{0}}\left(h_{1}^{\alpha} l_{\alpha}\right)=0, \\
\beta_{1}^{2} \frac{a}{r_{1}} \frac{a}{r_{0}}-\left(h_{1}^{\alpha} l_{\alpha}\right)\left(h_{0}^{\alpha} l_{\alpha}\right)=0 .
\end{aligned}
$$

Si nous mettons (19.1) sous la forme:

$$
\frac{\beta_{1}^{2} \frac{a}{r_{1}}}{\beta_{1}^{2} \frac{a}{r_{0}}}=\frac{h_{1}^{\alpha} l_{\alpha}}{h_{0}^{\alpha} l_{\alpha}}=\lambda
$$


il vient d'après (19.2):

$$
\lambda \alpha_{0}=0
$$

soit $\lambda=0$, ce qui est impossible. Il y a incompatibilité de l'onde de choc $\Sigma$ avec les ondes d'Alfven.

b) Supposons maintenant

Il en résulte:

$$
\alpha_{1}<0, \quad \alpha_{0}=0 .
$$

$$
\beta_{0} \frac{a}{r_{0}}+h_{0}^{\alpha} l_{\alpha}=0 .
$$

Avant le choc, une onde de type $B$ et une onde de type $A$ tangente à $\Sigma$ peuvent aboutir en $x \in \Sigma$ créant une perturbation arbitraire $\left(\delta u_{0}^{3}, \delta h_{0}^{3}\right)$.

Mais peuvent s'éloigner de $x$ l'onde de type $A$ pour l'état $Y_{0}$ portant une perturbation $\left(\delta u_{0 A}^{3}, \delta h_{0 A}^{3}\right)$ vérifiant

$$
\beta_{0} \delta u_{0 A}^{3}+\delta h_{0 A}^{3}=0
$$

et une onde d'Alfven de type $B$ pour l'état $Y_{1}$ portant une perturbation $\left(\delta u_{1 B}^{3}, \delta h_{1 B}^{3}\right)$ vérifiant:

$$
\beta_{1} \delta u_{1 B}^{3}-\delta h_{1 B}^{3}=0 .
$$

Les relations (18.3) relient cette dernière perturbation à une perturbation $\left(\bar{\delta} u_{0}^{3}, \bar{\delta} h_{0}^{3}\right)$ antérieur au choc $\Sigma$ avec:

$$
\left.\begin{array}{c}
\left(h_{1}^{\alpha} l_{\alpha}-\beta_{1} \frac{a}{r_{1}}\right) \delta u_{1 B}^{3}=\left(h_{0}^{\alpha} l_{\alpha}\right) \bar{\delta} u_{0}^{3}-\frac{a}{r_{0}} \bar{\delta} h_{0}^{3} \\
\beta_{1}\left(\beta_{1} \frac{a}{r_{1}}-h_{1}^{\alpha} l_{\alpha}\right) \delta u_{1 B}^{3}=\beta_{0}^{2} \frac{a}{r_{0}} \bar{\delta} u_{0}^{3}-\left(h_{0}^{\alpha} l_{\alpha}\right) \bar{\delta} h_{0}^{3} .
\end{array}\right\}
$$

Ainsi pour qu'une perturbation $\left(\bar{\delta} u_{0}^{3}, \bar{\delta} h_{0}^{3}\right)$ puisse être transmise à travers le choc $\Sigma$, il faut et il suffit que:

$$
\left(\beta_{1} h_{0}^{\alpha} l_{\alpha}+\beta_{0}^{2} \frac{a}{r_{0}}\right) \bar{\delta} u_{0}^{3}-\left(\beta_{1} \frac{a}{r_{0}}+h_{0}^{\alpha} l_{\alpha}\right) \bar{\delta} h_{0}^{3}=0 .
$$

Nous établirons dans un instant que

$$
\beta_{1} \frac{a}{r_{0}}+h_{0}^{\alpha} l_{\alpha} \neq 0 .
$$

L'onde de choc $\Sigma$ sera compatible avec la perturbation arbitraire $\left(\delta u_{0}^{3}, \delta h_{0}^{3}\right)$ s'il existe toujours une décomposition

$$
\left.\begin{array}{l}
\bar{\delta} u_{0}^{3}+\delta u_{0 A}^{3}=\delta u_{0}^{3} \\
\bar{\delta} h_{0}^{3}+\delta h_{0 A}^{3}=\delta h_{0}^{3}
\end{array}\right\}
$$

avec:

où l'on a posé:

$$
\bar{\delta} h_{0}^{3}=\Pi \bar{\delta} u_{0}^{3}, \quad \delta h_{0 A}^{3}=-\beta_{0} \delta u_{0 A}^{3}
$$

$$
I=\frac{\beta_{1} h_{0}^{\alpha} l_{\alpha}+\beta_{0}^{2} \frac{a}{r_{0}}}{\beta_{1} \frac{a}{r_{0}}+h_{0}^{\alpha} l_{\alpha}} .
$$


On est ainsi amené à étudier le système linéaire:

$$
\left.\begin{array}{rl}
\bar{\delta} u_{0}^{3}+\delta u_{0 A}^{3} & =\delta u_{0}^{3} \\
\Pi \bar{\delta} u_{0}^{3}-\beta_{0} \delta u_{0 A}^{3} & =\delta h_{0}^{3} .
\end{array}\right\}
$$

Le déterminant de (19.7) est donné par:

$$
\begin{gathered}
\Pi+\beta_{0}=\frac{\beta_{1} h_{0}^{\alpha} l_{\alpha}+\beta_{0} \frac{a}{r_{0}}+\beta_{0}\left(\beta_{1} \frac{a}{r_{0}}+h_{0}^{\alpha} l_{\alpha}\right)}{\beta_{1} \frac{a}{r_{0}}+h_{0}^{\alpha} l_{\alpha}}=\frac{\beta_{0}+\beta_{1}}{\beta_{1} \frac{a}{r_{0}}+h_{0}^{\alpha} l_{\alpha}} \\
\cdot\left(\beta_{0} \frac{a}{r_{0}}+h_{0}^{\alpha} l_{\alpha}\right)=0 .
\end{gathered}
$$

Ainsi (19.7) n'admet pas de solution pour toutes valeurs des seconds membres et $\Sigma$ n'est pas compatible avec les ondes d'Alfven.

Il nous reste à montrer que $\beta_{1} \frac{a}{r_{0}}+h_{0}^{\alpha} l_{\alpha} \neq 0$. Sinon on aurait $\beta_{1}=\beta_{0}$ ou

ce qui peut s'écrire:

$$
c^{2} r_{1} f_{1}+\mu\left|h_{1}\right|^{2}=c^{2} r_{0} f_{0}+\mu\left|h_{0}\right|^{2}
$$

$$
r_{1}^{2}\left(c^{2} \tau_{1}+\mu \frac{\left|h_{1}\right|^{2}}{r_{1}^{2}}\right)=r_{0}^{2}\left(c^{2} \tau_{0}+\mu \frac{\left|h_{0}\right|^{2}}{r_{0}^{2}}\right)
$$

ou

Il en résulte:

$$
r_{1}^{2}\left(\alpha_{1}+\mu \frac{\left(h_{1}^{\alpha} l_{\alpha}\right)^{2}}{a^{2}}\right)=r_{0}^{2} \mu \frac{\left(h_{0}^{\alpha} l_{\alpha}\right)^{2}}{a^{2}}=r_{0}^{2} \mu \frac{\left(h_{1}^{2} l_{2}\right)^{2}}{a^{2}} \frac{f_{1}^{2}}{f_{0}^{2}} .
$$

$$
\frac{1}{\tau_{1}^{2}} \alpha_{1}=\mu \frac{\left(h_{1}^{\alpha} l_{2}\right)^{2}}{a^{2}}\left(\frac{1}{\tau_{0}^{2}}-\frac{1}{\tau_{1}^{2}}\right)
$$

soit

Il vient

$$
\frac{\left(h_{1}^{\alpha} l_{\alpha}^{\prime}\right)^{2}}{a^{2}}\left(\tau_{0}+\tau_{1}\right)=\left(\frac{\left(h_{1}^{\alpha} l_{2}\right)^{2}}{a^{2}}-\frac{\left|h_{1}\right|^{2}}{r_{1}^{2}}\right) \tau_{0}
$$

$$
\frac{\left|h_{1}\right|^{2}}{r_{1}^{2}} \tau_{0}+\frac{\left(h_{1}^{\alpha} l_{\alpha}\right)^{2}}{a^{2}} \tau_{1}=0
$$

ce qui est contradictoire.

Nous pouvons énoncer pour un choc non tangentiel.

Théorème 1. Si $\Sigma$ est une onde de choc telle que $\alpha_{0} \alpha_{1}=0$, elle est incompatible avec les ondes d'Alfven à moins qu'elle ne corresponde à un choc d'Alfven $\left(\alpha_{0}=\alpha_{1}=0\right)$.

c) Nous avons démontré ailleurs [5] par des raisonnements analogues le théorème suivant.

Théorème 2. Pour qu'un choc non d'Alfven soit compatible avec les ondes d'Alfven, il faut et il suffit que $\alpha_{0} \alpha_{1}>0$.

Les chocs envisagés peuvent donc être décomposés en chocs lents tels que

12 Commun.math. Phys., Vol. 12

$$
\alpha_{1}<\alpha_{0}<0
$$


et chocs rapides tels que:

$$
0<\alpha_{1}<\alpha_{0} .
$$

De la relation $k_{0}^{2} \alpha_{0}^{2}=k_{1}^{2} \alpha_{1}^{2}$ il résulte que dans un choc lent, la grandeur du champ magnétique diminue, alors qu'elle augmente dans un choc rapide.

20. Vitesses des ondes de choc magnétohydrodynamiques

a) Supposons que l'état $Y$ du fluide varie comme précédemment sous les conditions $\left(\mathrm{C}_{1}\right),\left(\mathrm{C}_{2}\right),\left(\mathrm{C}_{3}\right),\left(\mathrm{C}_{4}\right), Z$ décrivant dans le plan $(\bar{q}, \tau)$ une demi-droite $\Delta$ de pente négative issue de $Z_{0}$ de telle sorte que:

$$
\bar{q}-\bar{q}_{0}=\frac{c^{2} a^{2}}{l^{\alpha} l_{\alpha}}\left(\tau-\tau_{0}\right), \quad \tau \leqq \tau_{0} .
$$

Le long de $A$, on a d'après (15.2) en considérant $S$ comme fonction de $\tau$

$$
\tau_{S}^{\prime} \propto \frac{d S}{d \tau}=\frac{P(l)}{a^{2} l^{\alpha} l_{\alpha}}
$$

Nous allons établir le lemme suivant:

Lemme. Sous les hypothèses de compressibilité $\left(H_{1}\right),\left(H_{2}\right)$, en tout point $Z_{s}$ de $\Delta$ où $S$ est stationnaire

On a en effet:

$$
\left(\frac{d^{2} S}{d \tau^{2}}\right)_{s}<0
$$

$$
\frac{d S}{d \tau}=S_{\tau}^{\prime}+S_{p}^{\prime} \frac{d p}{d \tau}=S_{\tau}^{\prime}+S_{p}^{\prime}\left(\frac{d \bar{q}}{d \tau}-\frac{1}{2} \mu \frac{d \chi}{d \tau}\right)
$$

soit :

$$
\frac{d S}{d \tau}=S_{\tau}^{\prime}+S_{p}^{\prime}\left(\frac{c^{2} a^{2}}{l^{\alpha} l_{\alpha}}-\frac{1}{2} \mu \frac{d \chi}{d \tau}\right)
$$

En dérivant (20.3) par rapport à $\tau$, il vient:

$$
\begin{aligned}
\frac{d^{2} S}{d \tau^{2}}=S_{\tau^{2}}^{\prime \prime}+2 S_{\tau p}^{\prime \prime}\left(\frac{c^{2} a^{2}}{l^{\alpha} l_{\alpha}}\right. & \left.-\frac{1}{2} \mu \frac{d \chi}{d \tau}\right)+S_{p^{2}}^{\prime \prime}\left(\frac{c^{2} a^{2}}{l^{\alpha} l_{\alpha}}-\frac{1}{2} \mu \frac{d \chi}{d \tau}\right)^{2} \\
& -S_{p}^{\prime} \cdot \frac{1}{2} \mu \frac{d^{2} \chi}{d \tau^{2}} .
\end{aligned}
$$

En un point $Z_{\mathrm{s}}$ où $S$ est stationnaire:

$$
\left(\frac{c^{2} a^{2}}{l^{\alpha} l_{\alpha}}-\frac{1}{2} \mu \frac{d \chi}{d \tau}\right)_{s}=-\left(\frac{S_{\tau}^{\prime}}{S_{p}^{\prime}}\right)_{s} .
$$

Il en résulte qu'en un tel point:

$$
\left(\frac{d^{2} S}{d \tau^{2}}\right)_{s}=\frac{1}{\left(S_{p}^{\prime}\right)_{s}^{\prime}}\left\{S_{\tau^{2}}^{\prime \prime}\left(S_{p}^{\prime}\right)^{2}-2 S_{p \tau}^{\prime \prime} S_{p}^{\prime} S_{\tau}^{\prime}+S_{p^{2}}^{\prime \prime}\left(S_{\tau}^{\prime}\right)^{2}\right\}_{s}-\frac{1}{2} \mu\left(S_{p}^{\prime} \frac{d^{2} \chi}{d \tau^{2}}\right)_{s} .
$$

Partons de le relation:

$$
\chi \alpha^{2}=L
$$

et dérivons la en $\tau$. Il vient en tenant compte de $d \alpha / d \tau=c^{2}$

$$
\frac{d \chi}{d \tau} \alpha+2 c^{2} \chi=0
$$


En dérivant une seconde fois, on a:

On en déduit:

$$
\frac{d^{2} \chi}{d \tau^{2}} \alpha+3 c^{2} \frac{d \chi}{d \tau}=0
$$

$$
\frac{d^{2} \chi}{d \tau^{2}} \alpha^{2}=6 c^{4} \chi=6 c^{4} k^{2}
$$

On obtient ainsi en posant $Q=\tau_{p^{2}}^{\prime \prime}-3 \mu c^{4}\left(k^{2} / \alpha^{2}\right) \tau_{p}^{\prime 3}$ et tenant compte de $(5.6)$ :

$$
\left(\frac{d^{2} S}{d \tau^{2}}\right)_{s}=-\left\{\frac{1}{\tau_{p}^{\prime 2} \tau_{s}^{\prime}} Q\right\}_{s}
$$

où le second membre est strictement négatif d'après $\left(H_{1}\right)$ et $\left(H_{2}\right)$, ce qui démontre le lemme.

b) Considérons un choc qui, au point $x$ de $\Sigma$, fait passer de $Z_{0}$ à $Z_{1}$. Le long de la demi-droite $\Delta$ issue du point représentatif de $Z_{0}$ et passant par le point représentatif de $Z_{1}$, on a :

$$
\frac{d \mathscr{H}}{d \tau}=2 f \Theta \frac{d S}{d \tau} .
$$

$\mathscr{H}$ est stationnaire en un point au moins $Z_{s}$ du segment $\left(Z_{0}, Z_{1}\right)$ et en ce point $S$ est aussi stationnaire. Du lemme il résulte que le point $Z_{s}$ est unique sur $\Delta$ et correspond pour $S(\tau)$ à un maximum strict sur $\Delta$. La variable $\tau$ décroissant le long de $\Delta$, on a :

$$
\begin{aligned}
& \frac{d S}{d \tau}<0 \quad \text { pour } \tau>\tau_{s} \quad \text { et en particulier pour } \tau=\tau_{0}, \\
& \frac{d S}{d \tau}>0 \quad \text { pour } \tau<\tau_{s} \quad \text { et en particulier pour } \tau=\tau_{1} .
\end{aligned}
$$

Soit $v^{\Sigma}$ la vitesse de $\Sigma$ par rapport au fluide. Nous l'affecterons d'un indice pour les états $Y_{0}$ et $Y_{1}$. La relation (9.7) peut s'écrire:

$$
\frac{a^{2}}{\left.\left(c^{2} r f+\mu|h|^{2}\right)\left(u^{\alpha} l_{\alpha}\right)^{2}-l^{\alpha} l_{\alpha}\right)} \alpha=\frac{\left(v^{\Sigma}\right)^{2}}{c^{2}}-\frac{\left(v^{A}\right)^{2}}{c^{2}} .
$$

$1^{\circ}$ Considérons d'abord un choc rapide $\left(0<\alpha_{1}<\alpha_{0}\right)$. D'après (20.4), on a :

$$
v_{0}^{\Sigma}>v_{0}^{A}, \quad v_{1}^{\Sigma}>v_{1}^{A} .
$$

De la relation (20.1) il résulte que $P(l)$ est positif avant le choc, négatif après et d'après (9.4) il en est de même pour $\Pi\left(y^{\Sigma}\right)$. On en déduit:

$$
v_{0}^{\Sigma}>v_{0}^{M R}, \quad v_{1}^{\Sigma}<v_{1}^{M R} .
$$

$2^{\circ}$ Considérons maintenant un choc lent $\left(\alpha_{1}<\alpha_{0}<0\right)$. D'après (20.4), on a:

$$
v_{0}^{\Sigma}<v_{0}^{A}, \quad v_{1}^{\Sigma}<v_{1}^{A}
$$


$\alpha$ étant négatif avant comme après le choc, il résulte de $(20.1)$ que $P(l)$ est négatif avant le choc, positif après. On en déduit de même

$$
v_{0}^{\Sigma}>v_{0}^{M L}, \quad v_{1}^{\Sigma}<v_{1}^{M L} .
$$

Nous pouvons résumer les résultats de cette étude dans l'énoncé suivant:

Théorème. Sous les hypothèses de compressibilité $\left(\mathrm{H}_{1}\right),\left(\mathrm{H}_{2}\right)$, les vitesses $v_{0}^{\Sigma}$ et $v_{1}^{\Sigma}$ par rapport au fluide d'une onde de choc magnétohydrodynamique respectivement avant et après le choc vérifient les inégalités suivantes.

1. Pour un choc rapide

$$
v_{0}^{M L}<v_{0}^{A}<v_{0}^{M R}<v_{0}^{\Sigma}, \quad v_{1}^{M L}<v_{1}^{A}<v_{1}^{\Sigma}<v_{1}^{M R} .
$$

2. Pour un choc lent

$$
v_{0}^{M L}<v_{0}^{\Sigma}<v_{0}^{A}<v_{0}^{M R}, \quad v_{1}^{\Sigma}<v_{1}^{M L}<v_{1}^{A}<v_{1}^{M R}
$$

où interviennent les vitesses magnétosoniques lentes et rapides et les vitesses d'Alfven avant et après le choc.

Considérons le cas de l'hydrodynamique relativiste $h^{\beta}=0$. Il n'y a pas de choc lent et l'on a $v^{A}=0, v^{M L}=0, v^{M R}=v$. Les inégalités (20.5) conduisent dans ce cas aux inégalités classiques (voir IsRaEL [3]).

c) Je démontrerai ailleurs que dans le voisinage de $Y_{0}$ :

$$
S-S_{0}=\left(\frac{Q}{12 f \Theta}\right)_{0}\left(p-p_{0}\right)^{3}, \quad\left(Q=\tau_{p^{2}}^{\prime \prime}-3 \mu c^{4} \frac{k^{2}}{\alpha^{2}} \tau_{p}^{\prime}>0\right) .
$$

L'accroissement d'entropie est ainsi du $3^{\circ}$ ordre par rapport à la puissance du choc $\left(p_{1}-p_{0}\right)[9]$.

\title{
Bibliographie
}

1. Choquet-Bruhat, Y.: Astron. Acta 6, 354-365 (1960).

2. Hofmmann, F., et E. Teller: Phys. Rev. 80, 692-702 (1950).

3. Israel, W.: Proc. Roy. Soc. A 259, 129-143 (1960).

4. Lichnerowicz, A.: Propagateurs et commutateurs en relativité générale. Publ. Math. Inst. Hautes Etudes Scient. Paris 1961.

5. - Ann. Inst. Henri Poincaré 5, 37-75 (1966).

6. - Relativistic hydrodynamics and magnetohydrodynamics. New-York: Benjamin 1967.

7. - Ann. Inst. Henri Poincaré 7, 271-302 (1967).

8. TaUb, A. H.: Phys. Rev. 74, 328-334 (1948).

9. Lichnerowicz, A.: Compt. Rend. 268, 256-260 (1969)

\author{
A. Lichnerowicz \\ Department of Physics \\ The Rockefeller University \\ New York, N. Y. 10021 USA \\ et Collège de France, F 75 Paris
}

